\title{
Structural Characterization of Polyglutamine Fibrils by Solid-State NMR Spectroscopy
}

\author{
Robert Schneider ${ }^{1}$, Miria C. Schumacher ${ }^{2}$, Henrik Mueller ${ }^{1}$, \\ Deepak Nand ${ }^{3}$, Volker Klaukien ${ }^{1}$, Henrike Heise ${ }^{1}$, Dietmar Riedel ${ }^{1}$, \\ Gerhard Wolf ${ }^{1}$, Elmar Behrmann ${ }^{2}$, Stefan Raunser ${ }^{2}$, Ralf Seidel ${ }^{2}$, \\ Martin Engelhard $^{2 \star}$ and Marc Baldus ${ }^{3 *}$
}

\author{
${ }^{1}$ Max Planck Institute for Biophysical Chemistry, Am Fassberg 11, 37077 Göttingen, Germany \\ ${ }^{2}$ Max Planck Institute for Molecular Physiology, Otto-Hahn-Straße 11, 44227 Dortmund, Germany \\ ${ }^{3}$ Bijooet Centre for Biomolecular Research, Utrecht University, Padualaan 8, $3584 \mathrm{CH}$ Utrecht, The Netherlands
}

Received 22 December 2010; received in revised form

21 June 2011;

accepted 27 June 2011

Available online

13 July 2011

\section{Edited by A. G. Palmer III}

Keywords:

amyloid fibrils;

Huntington's disease;

aggregation;

polyglutamine;

solid-state NMR
Protein aggregation via polyglutamine stretches occurs in a number of severe neurodegenerative diseases such as Huntington's disease. We have investigated fibrillar aggregates of polyglutamine peptides below, at, and above the toxicity limit of around 37 glutamine residues using solid-state NMR and electron microscopy. Experimental data are consistent with a dry fibril core of at least 70-80 $\AA$ in width for all constructs. Solid-state NMR dipolar correlation experiments reveal a largely $\beta$-strand character of all samples and point to tight interdigitation of hydrogen-bonded glutamine side chains from different sheets. Two approximately equally frequent populations of glutamine residues with distinct sets of chemical shifts are found, consistent with local backbone dihedral angles compensating for $\beta$-strand twist or with two distinct sets of side-chain conformations. Peptides comprising 15 glutamine residues are present as single extended $\beta$-strands. Data obtained for longer constructs are most compatible with a superpleated arrangement with individual molecules contributing $\beta$-strands to more than one sheet and an antiparallel assembly of strands within $\beta$-sheets.

(C) 2011 Elsevier Ltd. All rights reserved.

\footnotetext{
${ }^{*}$ Corresponding authors. E-mail addresses:

martin.engelhard@mpi-dortmund.mpg.de; m.baldus@uu.nl.

Present addresses: R. Schneider, Institut de Biologie Structurale, 41 rue Jules Horowitz, 38027 Grenoble, France; H. Mueller, Forschungszentrum Jülich, ICS 6, 52425 Jülich and Heinrich-Heine-Universität Düsseldorf, Universitätsstraße 1, 40225 Düsseldorf, Germany; H. Heise, Forschungszentrum Jülich, ICS 6, 52425 Jülich and Heinrich-Heine-Universität Düsseldorf,

Universitätsstraße 1, 40225 Düsseldorf, Germany.

Abbreviations used: CP, cross-polarization; EM, electron microscopy; FSLG, frequency-switched LeeGoldburg decoupling; HETCOR, heteronuclear correlation; MAS, magic angle spinning; ssNMR, solidstate NMR; Fmoc, 9-fluorenylmethyloxycarbonyl; TFA, trifluoroacetic acid.
}

\section{Introduction}

A variety of diseases involving proteins with expanded glutamine repeat stretches are known, including Huntington's disease and various types of spinocerebellar ataxia. ${ }^{1-4}$ While the proteins involved are usually unrelated except for the presence of polyglutamine regions, there is always a critical length of the glutamine stretch above which disease occurs, usually around 37 residues. ${ }^{2,4}$ Disease progression is correlated with the accumulation of neuronal intranuclear inclusions of amyloid fibrils formed by these proteins. $2,3,5$ Polyglutamine peptides alone can also form fibrils that bear the characteristic features of amyloid, that is, a linear and unbranched appearance in electron microscopy 
(EM), a cross- $\beta$ X-ray diffraction pattern, and staining by the dyes Congo red and thioflavin T. ${ }^{6}$ Polyglutamine aggregation kinetics and age of onset of Huntington's disease have been shown to correlate with the glutamine repeat length. ${ }^{7}$ However, toxicity has recently been attributed to monomeric or oligomeric polyglutamine species. ${ }^{8,9}$ Flanking sequences in the host protein have also been shown to influence kinetics and mechanisms of aggregation as well as toxicity of polyglutamine-containing proteins. ${ }^{10,11}$ Nevertheless, polyglutamine expansions are necessary for aggregation and toxicity of the respective proteins, and aggregation in all cases leads to amyloid fibrils with the polyglutamine stretch in the fibrillar core. ${ }^{10}$

In addition to the role of polyglutamine-containing proteins in human disease, regions rich in glutamine and asparagine have been found in a number of yeast prions. Here, they mediate aggregation to amyloid fibrils as a mechanism of epigenetic regulation and inheritance. ${ }^{12}$ Evidence also emerges for a role of glutamine- and asparagine-rich regions in proteinprotein interactions. ${ }^{13,94}$ While a large number of biochemical, structural, and molecular dynamics studies have investigated aggregates of proteins and peptides with polyglutamine stretches, their structural arrangement is still a matter of debate. Crystal structures of short asparagine- and glutamine-rich peptides related to amyloid fibrils have been reported, ${ }^{15,16}$ exhibiting extended strands in a parallel cross $-\beta$ arrangement with densely packed side chains. For longer peptides, various models have been proposed. Many of these involve extended $\beta$-strands of varying length, connected by short turns within the plane of a $\beta$-sheet or by longer $\beta$-bends between stacked sheets. ${ }^{17-20}$ The latter arrangement has also been postulated for glutamine- and asparagine-rich fragments of yeast prions. ${ }^{21,22}$ In contrast, Perutz et al. have developed a water-filled $\beta$-helix model believed to be in better agreement with some features of the fibril X-ray diffractogram. ${ }^{23}$ However, it has been shown that a $\beta$-hairpin structure with $\beta$-sheets stacked tightly on top of each other may account for these features as well. ${ }^{24}$ Moreover, molecular dynamics simulations have indicated that water-filled circular $\beta$-helix structures are unstable ${ }^{20,25}$ and suggested triangular $\beta$-helices instead, with inward-pointing side chains forming a dry fibril core. ${ }^{25}$ Some of these models also allow for parallel or antiparallel arrangement of $\beta$-strands within a sheet and between the sheets.

Solid-state NMR (ssNMR) allows for the investigation of insoluble, noncrystalline materials and has become a leading method for structure elucidation of amyloid fibrils (see Refs. 26-32). Here, we have used ssNMR and EM to study fibrils formed by polyglutamine peptides of varying length, below, at, and above the disease threshold. The peptides investigated comprised charged residues flanking the polyglutamine stretch, which has been shown to increase their solubility and slow down aggregation kinetics, while leaving fibril morphology unaffected. ${ }^{6}$ We have investigated both overall fibril arrangement and local structure by ssNMR and EM techniques, leading to a structural model of polyglutamine fibrils.

\section{Results}

\section{Electron microscopy}

Figure 1 shows electron micrographs of aggregates grown from the three peptides investigated. All of them form fibrillar aggregates whose length and degree of order appear to increase with the length of the construct. In agreement with EM data from an earlier study, ${ }^{6} \mathrm{D}_{2} \mathrm{Q}_{15} \mathrm{~K}_{2}$ does not form the highly ordered, long filaments that are characteristic for typical amyloid fibrils (Fig. 1a). Rather, smaller fibrillar aggregates of around $70 \AA$ in width and few hundreds of angstroms in length that cluster together laterally and longitudinally to form higher-order aggregates are visible. $\mathrm{GK}_{2} \mathrm{Q}_{38} \mathrm{~K}_{2}$ aggregates (Fig. 1b) are in the order of 100-200 nm in length, with a broader, ribbon-like appearance and widths up to about $200 \AA$, likely due to lateral clustering of individual protofilaments. Minimal fibril widths observed are around 70-80 $\AA$. $\mathrm{GK}_{2} \mathrm{Q}_{54} \mathrm{~K}_{2}$ forms fibers several hundred nanometers in length (Fig. 1c) that often exhibit an intertwined appearance. In this construct, various fibril widths up to about $160 \AA$ can be found, while the minimum width is again about $80 \AA$. In several samples and constructs, some additional unfibrillized protein is present.

The EM data agree well with earlier studies on length-dependent properties of polyglutamine aggregates, which indicated that only aggregates from peptides with a glutamine repeat length above the disease threshold of around 37 residues exhibit all features of classical amyloid fibrils. ${ }^{6}$ They also agree with observations that polyglutamine peptides of intermediate length (in the range of $20-40$ repeats) tend to form broader, more ribbon-like aggregates than longer constructs, which appear to assemble in a more typical amyloid-fibril-like fashion with diameters around $80 \AA .{ }^{6}$ Nevertheless, also the shorter peptides form fibrillar aggregates, which are known to exhibit a cross- $\beta$ X-ray diffraction pattern and bind thioflavin $\mathrm{T}$ similar to bona fide amyloid fibrils. ${ }^{6,23,24}$ The observed fibril widths indicate that the minimal fibrillar unit of polyglutamine aggregates is about 70 to $80 \AA$ wide. Thus, at least the longer constructs have to be folded instead of fully extended. The distribution of fibril widths in our samples indicates that there may be more than one 
(a)

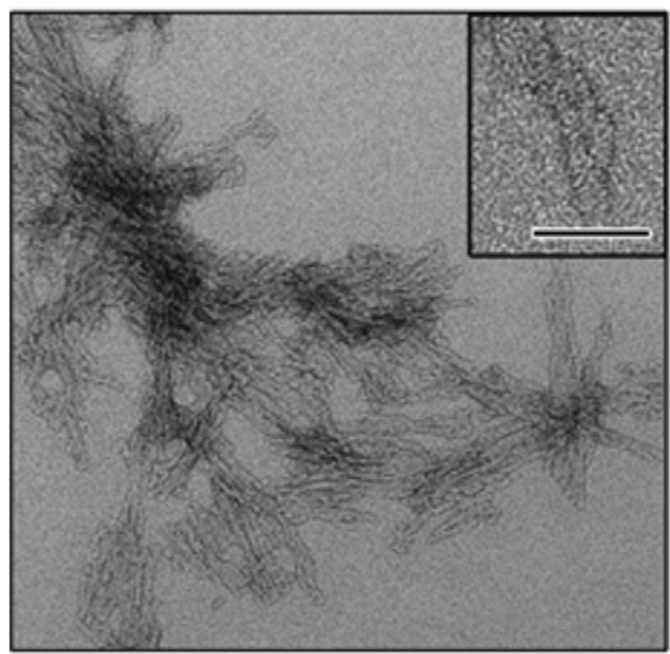

(b)

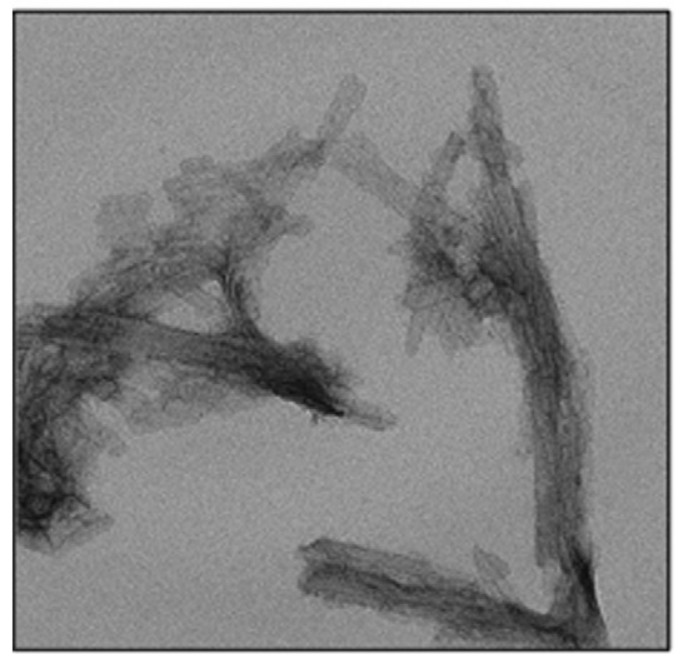

(c)

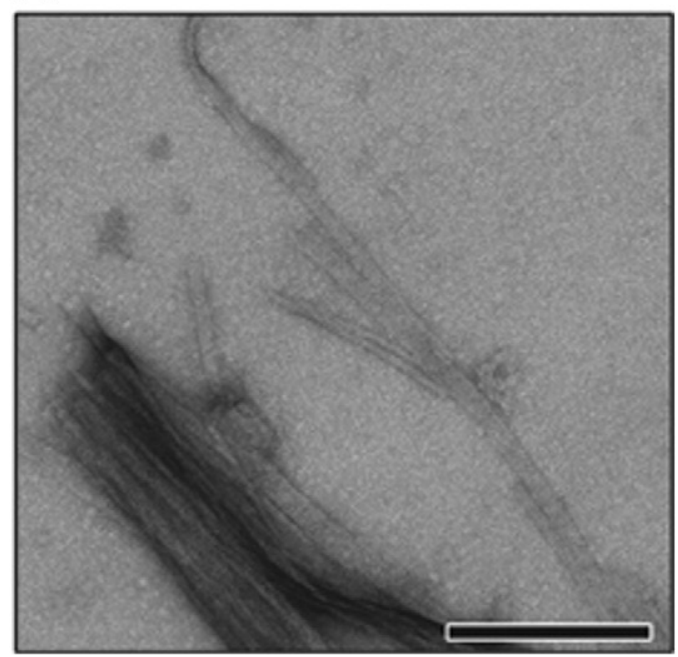

Fig. 1. Electron micrographs of $\mathrm{D}_{2} \mathrm{Q}_{15} \mathrm{~K}_{2}$ (a), $\mathrm{GK}_{2} \mathrm{Q}_{38} \mathrm{~K}_{2}$ (b), and $\mathrm{GK}_{2} \mathrm{Q}_{54} \mathrm{~K}_{2}$ (c) fibrillar aggregates. Scale bar in panel (c) represents $200 \mathrm{~nm}$, in inset $30 \mathrm{~nm}$. (a)

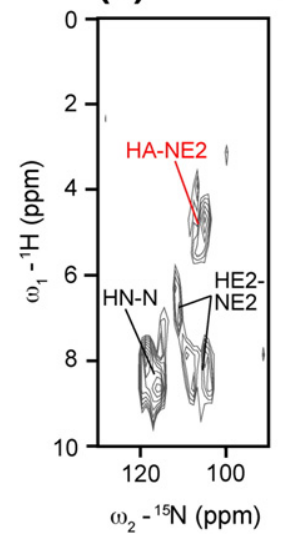

(b)

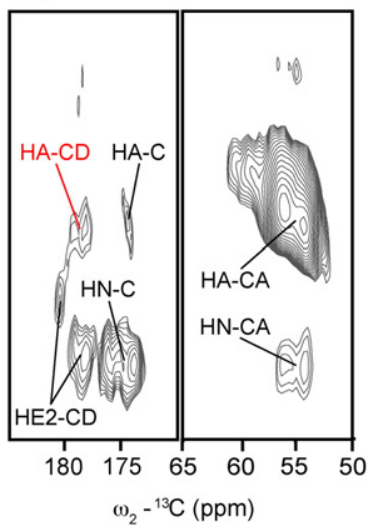

Fig. 2. FSLG-HETCOR $\left({ }^{1} \mathrm{H}^{15} \mathrm{~N}\right)$ (a) and $\left({ }^{1} \mathrm{H}^{13} \mathrm{C}\right)$ (b) correlation spectra of $\mathrm{GK}_{2} \mathrm{Q}_{38} \mathrm{~K}_{2}$. The correlations between glutamine nuclei giving rise to the individual cross peaks are indicated in the labels. Red labels denote likely intersheet or intermolecular correlations (see the text).

overall fold accessible to polyglutamine monomers within fibrils, apart from different levels of lateral aggregation of protofilaments.

\section{Overall fibril organization}

We initially conducted ssNMR experiments to investigate the overall arrangement of polyglutamine fibrils. In order to probe hydrogen-bonding interactions, we performed ${ }^{1} \mathrm{H}-{ }^{15} \mathrm{~N}$ and ${ }^{1} \mathrm{H}-{ }^{13} \mathrm{C}$ heteronuclear correlation (HETCOR) correlation experiments on $\mathrm{GK}_{2} \mathrm{Q}_{38} \mathrm{~K}_{2}$ fibrillar aggregates under proton decoupling [frequency-switched LeeGoldburg decoupling (FSLG) ${ }^{33}$ (Fig. 2). At the short cross-polarization (CP) times used, one- and twobond correlations from rigid fibril regions dominate the spectrum. Interestingly, a major fraction of glutamine side-chain $\mathrm{H}^{\varepsilon 2 \mathrm{P} / 22}$ proton resonances are found shifted downfield to $8 \mathrm{ppm}$ and more,

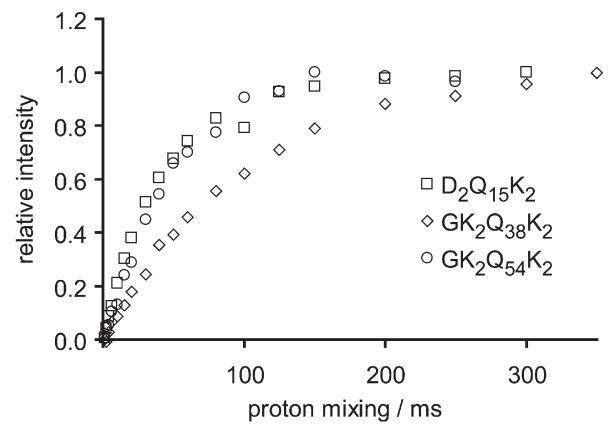

Fig. 3. Buildups of water-edited $\mathrm{CP}$ intensity in the aliphatic spectral region for the three polyglutamine constructs (open symbols). 


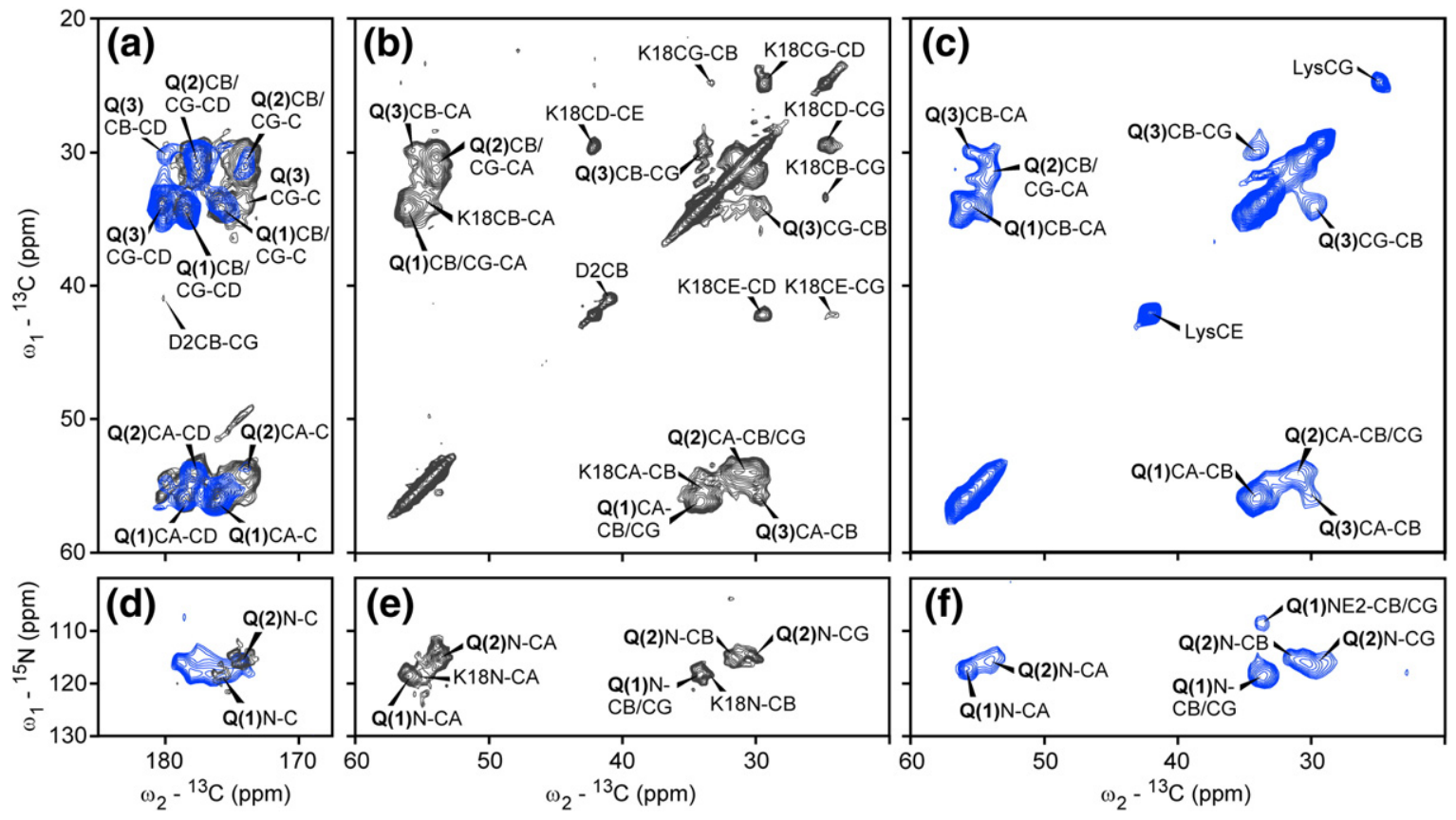

Fig. 4. Intra-residue correlation spectra. $(\mathrm{a}-\mathrm{c})\left({ }^{13} \mathrm{C},{ }^{13} \mathrm{C}\right)$ spin diffusion correlation spectra (150 ms mixing time) of $\mathrm{D}_{2} \mathrm{Q}_{15} \mathrm{~K}_{2}$ (black) and $\mathrm{GK}_{2} \mathrm{Q}_{54} \mathrm{~K}_{2}$ (blue). (a) shows an overlay of the carbonyl region of both constructs, while (b) and (c) display the aliphatic regions individually. Bracketed numbers in assignments refer to the different glutamine populations (see the text). (d-f) Intra-residue NCACB correlation spectra of the same two constructs, with color code as in (a) to (c). Note the longer $\left({ }^{13} \mathrm{C},{ }^{13} \mathrm{C}\right)$ mixing time used in the $\mathrm{GK}_{2} \mathrm{Q}_{54} \mathrm{~K}_{2}$ spectrum $\left[\mathrm{blue}\right.$, (d) and (f)] after the $\left({ }^{15} \mathrm{~N},{ }^{13} \mathrm{C}\right)$ transfer step, explaining the correlations of the backbone amide nitrogen with side-chain carbonyl resonances for $\mathrm{GK}_{2} \mathrm{Q}_{54} \mathrm{~K}_{2}$ in (d).

indicative of side-chain hydrogen-bonding interactions. ${ }^{34-36}$ In addition, cross peaks that can only be explained by interactions between backbone $\mathrm{H}^{\alpha}$ protons and side-chain $\mathrm{N}^{\varepsilon 2}$ and $\mathrm{C}^{\delta}$ resonances are found (red labels in Fig. 2). These observations strongly speak in favor of a tight interdigitation of side chains from adjacent $\beta$-sheets that brings sidechain termini of one $\beta$-sheet and the $\beta$-strand backbone of the next sheet into close contact, as proposed by "steric zipper" models of fibril structure. ${ }^{15,24}$ Such interactions may also account for the considerable deviation of ${ }^{15} \mathrm{~N}$ side-chain chemical shifts from their average value in the Biological Magnetic Resonance Data Bank. ${ }^{37}$

To complement these data, we performed wateredited ssNMR experiments (see Refs. 38-40) that provide information about the water accessibility of biomolecules and polymers. Data from water-edited buildup experiments on the three polyglutamine constructs are shown in Fig. 3 and Table 1 in Supporting Information. Assuming compact, dry fibrils with no water inside, our analysis (see Supporting Information) yields average fibril widths of about $70 \AA$ for $D_{2} Q_{15} K_{2}, 110 \AA$ for $\mathrm{GK}_{2} \mathrm{Q}_{38} \mathrm{~K}_{2}$, and $85 \AA$ for $\mathrm{GK}_{2} \mathrm{Q}_{54} \mathrm{~K}_{2}$. These values correspond well to the widths most commonly found in electron micrographs and are in line with the different width distributions and levels of lateral aggregation seen in EM. In contrast, a water-filled fibril structure ${ }^{23}$ yielding the same buildup characteristics in ssNMR experiments would require a fibril width much larger than seen in EM images.

Table 1. List of average chemical shifts (in parts per million) observed for the three glutamine populations across the different polyglutamine samples investigated

\begin{tabular}{|c|c|c|c|c|c|c|c|c|}
\hline $\begin{array}{l}\text { Gln population or } \\
\mathrm{D}_{2} \mathrm{Q}_{15} \mathrm{~K}_{2} \text { residue }\end{array}$ & $\mathrm{N}$ & $\mathrm{C}$ & CA & $\mathrm{CB}$ & CG & CD & $\mathrm{CE}$ & NE2 \\
\hline $\mathrm{Q}(1)$ & 118.0 & 175.9 & 55.9 & 34.2 & 33.9 & 178.5 & & 107.9 \\
\hline $\mathrm{Q}(2)$ & 115.4 & 174.0 & 54.0 & 31.6 & 29.8 & 177.4 & & 104.4 \\
\hline $\mathrm{Q}(3)$ & & 174.0 & 55.7 & 29.9 & 33.9 & 180.2 & & \\
\hline D2 & & 177.6 & 54.6 & 40.9 & 180.0 & & & \\
\hline K18 & 118.6 & 174.8 & 55.1 & 33.5 & 24.7 & 29.5 & 42.1 & \\
\hline
\end{tabular}

Glutamine populations $\mathrm{Q}(1)$ and $\mathrm{Q}(2)$ constitute $\beta$-sheets; glutamine population $\mathrm{Q}(3)$ corresponds to residues in turn regions or unstructured molecules. For completeness, assignments for residues Asp2 and Lys18 of $\mathrm{D}_{2} \mathrm{Q}_{15} \mathrm{~K}_{2}$ are also given. 
Thus, our data favor a model in which polyglutamine fibrils have a dry, tightly packed fibrillar core similar to that of other types of amyloid. ${ }^{15,27,29-31,41}$

\section{Residue-specific ssNMR analysis}

Two-dimensional $\left({ }^{13} \mathrm{C}^{13} \mathrm{C}\right)$ ssNMR spectra of $\mathrm{D}_{2} \mathrm{Q}_{15} \mathrm{~K}_{2}, \mathrm{GK}_{2} \mathrm{Q}_{38} \mathrm{~K}_{2}$, and $\mathrm{GK}_{2} \mathrm{Q}_{54} \mathrm{~K}_{2}$ fibrillar aggregates reveal striking similarities indicative of a common structural organization. In Fig. 4, spectra are shown for the $\mathrm{Q}_{15}$ (black) and $\mathrm{Q}_{54}$ (blue) constructs. Most prominently, in all constructs and across different types of spectra selecting for more rigid parts of the sample, two major populations of glutamine residues whose resonance signals fall into distinct spectral regions are apparent [from now on referred to as $Q(1)$ and $Q(2)]$. These two main populations of glutamine residues appear in ssNMR spectra with approximately equal intensities in all constructs, suggesting a distribution close to 1:1. Resonances of all $\mathrm{Q}(1)$ nuclei are consistently shifted to higher frequencies with respect to $Q(2)$ resonances (Table 1), an observation previously also made for a glutamine residue in fibrils formed by an asparagine- and glutamine-rich peptide from the yeast prion Sup $35 .{ }^{42}$ Compared to average $C^{\alpha}$ and $\mathrm{C}^{\beta}$ chemical shift values for glutamine, both populations exhibit a strongly negative secondary chemical shift of about $-5 \mathrm{ppm}$, indicating that both groups of glutamine residues participate in $\beta$-sheet secondary structure. ${ }^{43}$ Polyglutamine fibrillar aggregates are hence largely composed of $\beta$-sheets, consistent with their cross- $\beta$ X-ray diffraction pattern. Notably, we also found cross peaks corresponding to $Q(1)$ and $Q$ (2) glutamines in the spectra of lyophilized polyglutamine samples after addition of water or buffer solution (Fig. 1, Supporting Information), even though no fibrils but only small spherical or elongated aggregates can be detected in such samples by EM (Fig. 2, Supporting Information). Thus, the local organization leading to the occurrence of two distinct glutamine populations appears to form independent of or before bona fide fibril formation. Compared to ssNMR spectra of other amyloid fibrils, the spectral linewidths of around $2 \mathrm{ppm}$ in ${ }^{13} \mathrm{C}$ and $3 \mathrm{ppm}$ in ${ }^{15} \mathrm{~N}$ dimensions indicate structural heterogeneity in all samples, consistent with ssNMR data on fibrils from the asparagine- and glutamine-rich protein Ure2p. ${ }^{21}$ Apparently, glutamine-rich sequences can assume slightly different molecular conformations within fibrillar aggregates, as also suggested by the distribution of fibril widths observed in EM for our $\mathrm{Q}_{38}$ and $\mathrm{Q}_{54}$ constructs.

Additionally, a third glutamine population $[Q(3)]$ is seen in $\left({ }^{13} \mathrm{C}_{1}^{13} \mathrm{C}\right)$ correlation spectra at longer mixing times, likely due to enhanced mobility in these residues. The corresponding $Q(3) C^{\alpha}$ and $C^{\beta}$ chemical shifts are close to random-coil values. These residues could thus constitute turns or unstructured regions within the fibrils or occur in unstructured, immobilized monomers. Evidence for even larger molecular mobility within polyglutamine fibrils was seen in a complementary set of scalar-based ssNMR experiments ${ }^{44}$ (Fig. 3, Supporting Information) for $\mathrm{GK}_{2} \mathrm{Q}_{38} \mathrm{~K}_{2}$ and $\mathrm{GK}_{2} \mathrm{Q}_{54} \mathrm{~K}_{2}$ constructs. Compared to CP-type ssNMR experiments that select for rigid regions, lysine signals are strongly enhanced with respect to glutamine signals in scalar-based spectra, indicating that chiefly the lysine-containing $\mathrm{N}$ - and C-termini of otherwise rigid molecules are represented in these spectra. These data suggest that the $\mathrm{N}$ - and C-termini of the longer polyglutamine constructs are not part of the fibril core.

Spectral resolution, uniform isotope labeling, and the dominant presence of only one residue type preclude residue-specific sequential resonance assignments in the longer polyglutamine constructs. In the selectively labeled $\mathrm{D}_{2} \mathrm{Q}_{15} \mathrm{~K}_{2}$ construct, on the other hand, sequential peaks from three residue pairs can be expected (Fig. 5a). The labeled residues Asp2 and Lys18 can be identified unambiguously in the spectra based on their chemical shifts, although Asp2 resonances are usually weak (Fig. 4b). The strongest peaks in the NCOCA $\left(\mathrm{N}_{i}, \mathrm{C}_{i-1}\right)$ correlation spectrum of $\mathrm{D}_{2} \mathrm{Q}_{15} \mathrm{~K}_{2}$ (Fig. 5a) correspond to correlations between two $\mathrm{Q}(2)$ residues as well as to $Q(2)-D 2$ and $K 18-Q(1)$ correlations. These findings, along with the labeling pattern of our $\mathrm{Q}_{15}$ construct, suggest that $\mathrm{Gln} 17$ belongs to glutamine population $\mathrm{Q}(1)$ and $\mathrm{G} \ln 3, \mathrm{G} \ln 9$, and $\mathrm{G} \ln 10$ belong to glutamine population $Q(2)$. However, smaller peaks in the NCOCA spectrum reveal that all these residues may, in principle, belong to either the $Q(1)$ or $\mathrm{Q}(2)$ population, and the $\mathrm{K} 18-\mathrm{Q}(1)$ correlation may also contain a contribution from two sequential $\mathrm{Q}(1)$ glutamines (i.e., $\mathrm{G} \ln 9$ and $\mathrm{G} \ln 10)$. These results indicate that the different chemical shifts of $Q(1)$ and $\mathrm{Q}(2)$ glutamines reflect local geometry and not necessarily specific positions within the peptide sequence. Importantly, the main signals in the $\mathrm{D}_{2} \mathrm{Q}_{15} \mathrm{~K}_{2}$ NCOCA spectrum do not contain a sequential correlation between $\mathrm{Q}(1)$ and $\mathrm{Q}(2)$ glutamines, and the sequential correlation between two $Q$ (2) glutamines [labeled $\mathrm{Q}(2) \mathrm{N}-\mathrm{CA}$ in Fig. 5a] can only be explained by the Gln9-Gln10 residue pair. This means that two consecutive central glutamines exhibit chemical shifts corresponding to an extended $\beta$-sheet conformation; that is, they cannot form a bend or a turn.

In line with the $\mathrm{Q}_{15}$ case, sequential NCOCA $\left(\mathrm{N}_{i}\right.$, $\mathrm{C}_{i-1}$ ) correlation spectra of the longer constructs (Fig. $5 b$ and c) exhibit spectral intensity predominantly at the same positions as seen in intra-residue NCACB $\left(\mathrm{N}_{i}, \mathrm{C}_{i}\right)$ correlation spectra, suggesting that glutamine residues of the two major $\beta$-sheet populations are mostly flanked by residues of the same population within the amino acid sequence. However, sequential correlations between $Q(1)$ and 

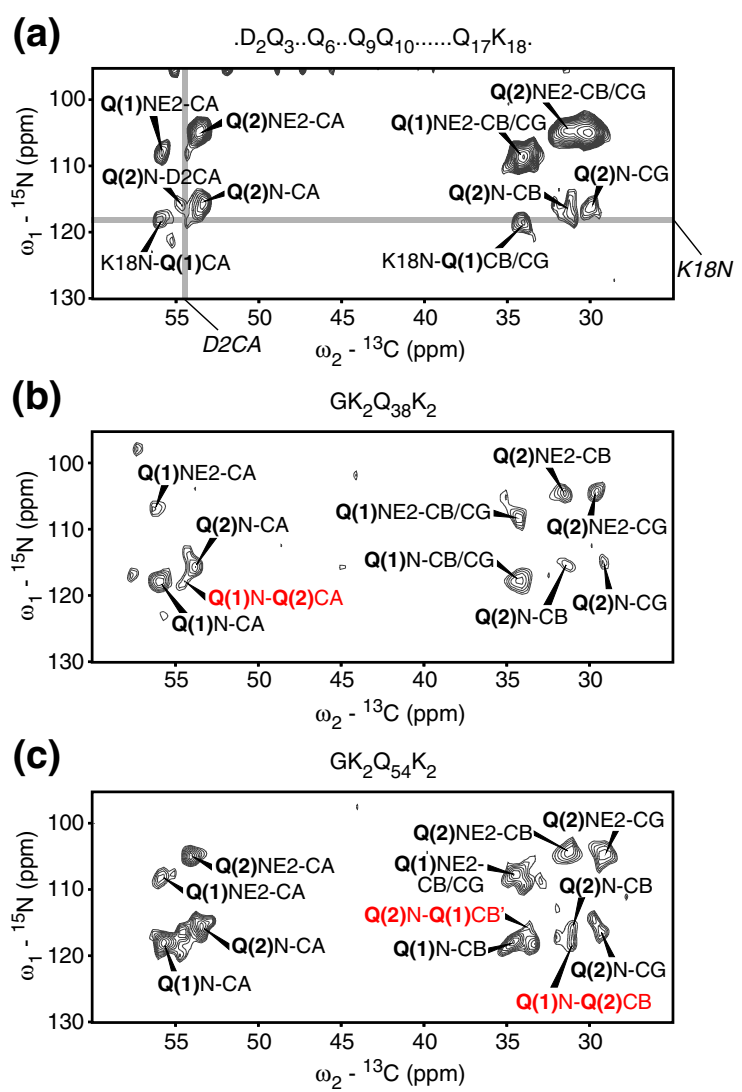

(d)

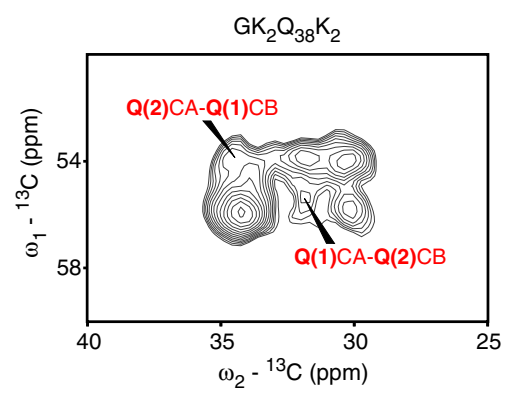

Fig. 5. Inter-residue correlation spectra. (a-c) Interresidue $\left(\mathrm{N}_{i}, \mathrm{C}_{i-1}\right)$ NCOCA correlation spectra of (a) $\mathrm{D}_{2} \mathrm{Q}_{15} \mathrm{~K}_{2}$, (b) $\mathrm{GK}_{2} \mathrm{Q}_{38} \mathrm{~K}_{2}$, and (c) $\mathrm{GK}_{2} \mathrm{Q}_{54} \mathrm{~K}_{2}$. Gray lines in (a) indicate chemical shifts of Lys18 $\mathrm{N}$ and Asp2 $\mathrm{C}^{\alpha}$ important for assignment; their most likely correlation partners are labeled. Red assignments in (b) and (c) indicate sequential correlations between different glutamine populations. (d) Section representing $C^{\alpha}-C^{\beta}$ correlations of a sequential $\left({ }^{13} \mathrm{C},{ }^{13} \mathrm{C}\right)$ spin diffusion spectrum of $\mathrm{GK}_{2} \mathrm{Q}_{38} \mathrm{~K}_{2}$ recorded under weak coupling conditions with a $150 \mathrm{~ms}$ mixing time. Sequential correlations between different glutamine populations are indicated.

$\mathrm{Q}(2)$ residues exist in $\mathrm{GK}_{2} \mathrm{Q}_{38} \mathrm{~K}_{2}$ and $\mathrm{GK}_{2} \mathrm{Q}_{54} \mathrm{~K}_{2}$, as also confirmed by $\left({ }^{13} \mathrm{C},{ }^{13} \mathrm{C}\right)$ correlation spectra recorded under weak coupling conditions ${ }^{45}$ (Fig. $5 d)$. Thus, glutamine residues of different chemical shift populations do occur within the same monomer but appear to be clustered in stretches of several residues of one population, as seen from sequential $\left(\mathrm{N}_{i}, \mathrm{C}_{i-1}\right)$ correlation spectra.

\section{Through-space correlations}

In order to probe intramolecular and intermolecular through-space contacts, we recorded N/ $\mathrm{CHHC}^{46,47}$ spectra and $\left({ }^{13} \mathrm{C},{ }^{13} \mathrm{C}\right)$ spin diffusion spectra with long mixing times (Fig. 6). A variety of cross peaks between different glutamine populations are readily seen and indicated in red. For the selectively labeled $\mathrm{D}_{2} \mathrm{Q}_{15} \mathrm{~K}_{2}$ sample, an NHHC spectrum with a $\left({ }^{1} \mathrm{H},{ }^{1} \mathrm{H}\right)$ mixing time of $100 \mu$ s is depicted in Fig. 6a (upper panel) in black, overlaid with the same spectrum recorded on a $\mathrm{D}_{2} \mathrm{Q}_{15} \mathrm{~K}_{2}$ sample diluted in unlabeled material before fibrillization (blue). Peaks observable only in the spectrum of the undiluted sample consequently correspond to intermolecular contacts. While the relatively short $\left({ }^{1} \mathrm{H},{ }^{1} \mathrm{H}\right)$ mixing time of $100 \mu$ s leads to the dominant presence of intra-residue and sequential correlations, ${ }^{48}$ cross peaks that are not present in NCACB or NCOCA correlation spectra or in the NHHC spectrum of diluted $\mathrm{D}_{2} \mathrm{Q}_{15} \mathrm{~K}_{2}$ can be identified. Already at this mixing time, weak intermolecular correlations between side-chain amide nitrogens of one glutamine population and sidechain as well as backbone carbons of the respective other glutamine population become apparent. This observation points to a well-ordered, rather rigid arrangement of glutamine side chains in the fibrillar aggregates. Moreover, the distance between a sidechain amide proton and a backbone $\mathrm{C}^{\alpha}$ proton in a neighboring strand within the same $\beta$-sheet should be on the order of 5-6 $\AA$ (assuming extended side chains and a standard interstrand separation of $4.8 \AA$ A) and would thus most likely be too long to be observed with the short $\left({ }^{1} \mathrm{H}^{1} \mathrm{H}\right)$ mixing time employed here. ${ }^{47}$ These correlations are thus best explained by a close proximity of glutamine sidechain amides and the next $\beta$-sheet layer stacked above or below. Together with the observed side chain-backbone correlations in FSLG-HETCOR spectra (Fig. 2), these results support the presence of tightly stacked $\beta$-sheets with interdigitated side chains in polyglutamine aggregates, as proposed by steric zipper models. ${ }^{15,24}$

In addition, a peak in the backbone amide nitrogen region confirms intermolecular contacts within the $\beta$-sheet plane between $Q(1)$ and $Q(2)$ glutamines or between Lys18 and a Q(2) glutamine. Although the labeled glutamine residues in $\mathrm{D}_{2} \mathrm{Q}_{15} \mathrm{~K}_{2}$ cannot unambiguously be assigned to the two shift populations, the main inter-residue NCOCA cross peaks suggest that $G \ln 3, G \ln 9$, and $G \ln 10$ mainly belong to the $\mathrm{Q}(2)$ population and $\mathrm{G} \ln 17$ mainly belongs to the $\mathrm{Q}(1)$ population. This intermolecular cross peak thus gives an indication that $\mathrm{D}_{2} \mathrm{Q}_{15} \mathrm{~K}_{2}$ monomers may assemble into fibrils in an 

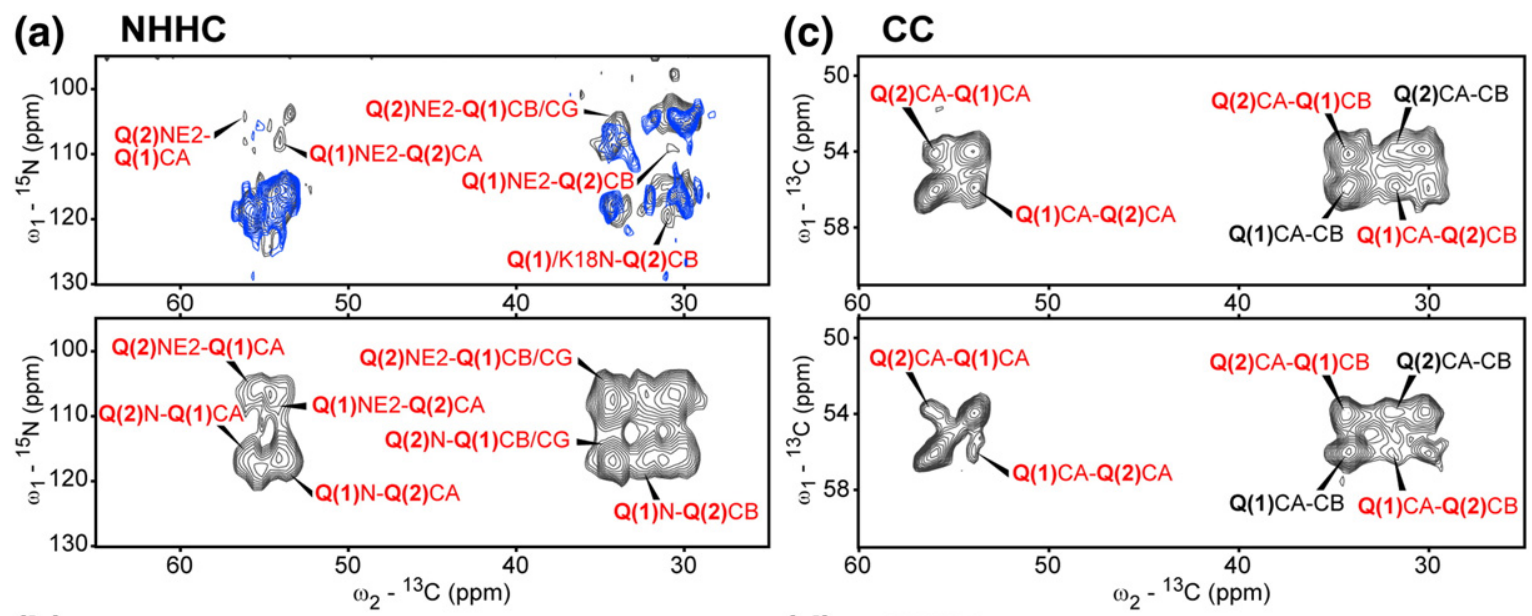

(b)

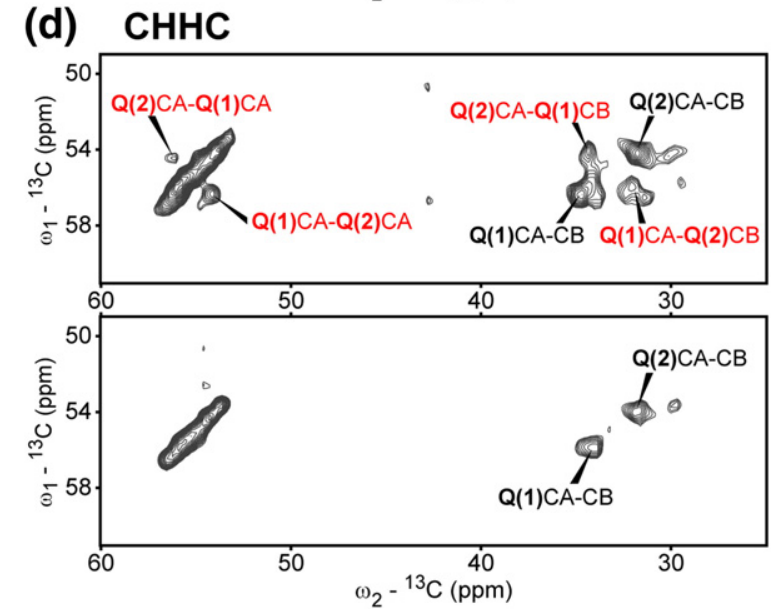

Fig. 6. Long-range and through-space correlations. Cross peaks between different glutamine populations are labeled in red. (a) Top: overlay of NHHC spectra (100 $\mu$ s mixing time) of undiluted, selectively labeled $\mathrm{D}_{2} \mathrm{Q}_{15} \mathrm{~K}_{2}$ fibrils (black) and selectively labeled $\mathrm{D}_{2} \mathrm{Q}_{15} \mathrm{~K}_{2}$ diluted in 2.5 parts of unlabeled material before fibrillization (blue). Bottom: NHHC spectrum (250 $\mu$ s mixing time) of uniformly ${ }^{15} \mathrm{~N}_{1}^{13} \mathrm{C}$-labeled $\mathrm{GK}_{2} \mathrm{Q}_{54} \mathrm{~K}_{2}$ fibrils. (b) Scheme illustrating the contacts seen in NHHC spectra in a hypothetical arrangement of stacked antiparallel $\beta$-sheets. (c) Cutouts from $\left({ }^{13} \mathrm{C}^{13} \mathrm{C}\right)$ spin diffusion spectra with a 500 ms mixing time of uniformly ${ }^{15} \mathrm{~N},{ }^{13} \mathrm{C}$-labeled $\mathrm{GK}_{2} \mathrm{Q}_{38} \mathrm{~K}_{2}$ fibrils (top) and of $\mathrm{GK}_{2} \mathrm{Q}_{38} \mathrm{~K}_{2}$ fibrils consisting of a $1: 1$ mixture of ${ }^{15} \mathrm{~N}$ - or ${ }^{13} \mathrm{C}$-labeled monomers (bottom). (d) Cutouts from CHHC spectra (250 $\mu$ s mixing time) recorded on uniformly ${ }^{15} \mathrm{~N}$, ${ }^{13} \mathrm{C}$-labeled $\mathrm{GK}_{2} \mathrm{Q}_{38} \mathrm{~K}_{2}$ fibrils (top) and on $\mathrm{GK}_{2} \mathrm{Q}_{38} \mathrm{~K}_{2}$ fibrils consisting of a $1: 1$ mixture of ${ }^{15} \mathrm{~N}$ - or ${ }^{13} \mathrm{C}$ labeled monomers (bottom).

antiparallel fashion. An NHHC spectrum with a longer mixing time of $250 \mu \mathrm{s}$, recorded on uniformly ${ }^{15} \mathrm{~N},{ }^{13} \mathrm{C}$-labeled $\mathrm{GK}_{2} \mathrm{Q}_{54} \mathrm{~K}_{2}$ fibrils, is depicted in the lower panel of Fig. 6a. Here, the through-space correlations between different glutamine populations are equally well represented as the correlations within individual residues or populations. Especially the side chain-backbone correlations between the populations further confirm a tight stacking of $\beta$ sheets as assumed in steric zipper models. Figure $6 \mathrm{~b}$ illustrates the NHHC contacts seen in the spectra of Fig. $6 \mathrm{a}$ in a hypothetical arrangement of closely stacked antiparallel $\beta$-sheets.

Further constraints on the orientation of polyglutamine monomers in fibrils were obtained from $\left({ }^{13} \mathrm{C}^{13} \mathrm{C}\right)$ spin diffusion and $\mathrm{CHHC}$ spectra recorded on $\mathrm{GK}_{2} \mathrm{Q}_{38} \mathrm{~K}_{2}$ and $\mathrm{GK}_{2} \mathrm{Q}_{54} \mathrm{~K}_{2}$ fibrils. In order to differentiate between intramolecular and intermolecular correlations, we compared the spectra of uniformly ${ }^{15} \mathrm{~N},{ }^{13} \mathrm{C}$-labeled fibrils with the spectra of fibrils prepared from a 1:1 mixture of either ${ }^{15} \mathrm{~N}$ or ${ }^{13} \mathrm{C}$-labeled monomers. Figure $6 \mathrm{c}$ shows a comparison of $\left({ }^{13} \mathrm{C}^{13} \mathrm{C}\right)$ spin diffusion spectra recorded with a long mixing time of $500 \mathrm{~ms}$ on uniformly ${ }^{15} \mathrm{~N},{ }^{13} \mathrm{C}$-labeled (top) and mixed ${ }^{15} \mathrm{~N}$ - or ${ }^{13} \mathrm{C}$-labeled $\mathrm{GK}_{2} \mathrm{Q}_{38} \mathrm{~K}_{2}$ fibrils (bottom). Correlations between glutamine residues of populations $\mathrm{Q}(1)$ and $Q(2)$ are strong in the uniformly labeled sample and attenuated with respect to the intrapopulation cross peaks in the mixed-labeled sample. This observation shows that the interpopulation cross peaks contain an intermolecular contribution. In order to clearly differentiate between intermolecular contributions and magnetization transfer along the carbon-carbon 
bond network dominant in $\left({ }^{13} \mathrm{C},{ }^{13} \mathrm{C}\right)$ spin diffusion spectra, ${ }^{46,47}$ we recorded $\mathrm{CHHC}$ spectra with a $250 \mu \mathrm{s}$ mixing time on the $\mathrm{GK}_{2} \mathrm{Q}_{38} \mathrm{~K}_{2}$ samples (Fig. 6d). Whereas correlations between glutamine populations 1 and 2 are clearly visible in the uniformly labeled sample, they are virtually absent in the mixed-labeled sample. This indicates that these cross peaks originate from intermolecular contacts and is consistent with our previous finding that most sequential contacts occur between glutamine residues of the same population. Since the distance between backbone atoms of residues in stacked sheets should not be smaller than about $8 \AA{ }^{24}$ it can be assumed that especially $C^{\alpha}-C^{\alpha}$ contacts between different populations occur chiefly between neighboring strands within the same $\beta$-sheet. The fact that they are not visible in the spectrum of the mixedlabeled sample strongly suggests that $\mathrm{GK}_{2} \mathrm{Q}_{38} \mathrm{~K}_{2}$ does not fold back within one $\beta$-sheet as an antiparallel hairpin. Individual monomers rather appear to fold in a superpleated $\beta$-sandwich fashion ${ }^{49}$ where one molecule contributes strands to different $\beta$-sheets stacked on top of each other. In this arrangement, all contacts between strands within one sheet are intermolecular, consistent with our $\mathrm{CHHC}$ data.

In addition, one of the $\mathrm{CHHC}$ cross peaks between $\mathrm{C}^{\alpha}$ nuclei of different glutamine populations appears with an intensity comparable to that of intraresidue or intrapopulation cross peaks in the spectrum of uniformly labeled $\mathrm{GK}_{2} \mathrm{Q}_{38} \mathrm{~K}_{2}$ (Fig. 6d, upper panel). This suggests that $\beta$-sheets in $\mathrm{GK}_{2} \mathrm{Q}_{38} \mathrm{~K}_{2}$ fibrils are mainly arranged in an antiparallel fashion, since the distance between $\mathrm{H}^{\alpha}$ protons in neighboring strands is much shorter in antiparallel $\beta$-sheets (about $2.3 \AA$ ) than in parallel sheets (about $4.8 \AA$ )..$^{50}$ Data on $\mathrm{GK}_{2} \mathrm{Q}_{54} \mathrm{~K}_{2}$ fibrils confirm these conclusions (Fig. 4, Supporting Information).

\section{A model of polyglutamine fibril structure}

Our data suggest that the polyglutamine fibrils investigated consist of mainly antiparallel $\beta$-sheets with glutamine side chains hydrogen bonded along the fibril axis within a sheet and tightly interdigitated between the sheets, forming a steric zipper. Monomers of the longer constructs appear to be folded back between different sheets that are stacked on top of each other in a superpleated arrangement. EM and water-edited ssNMR data point to a dry fibril core with a width of at least 70$80 \AA$ in all constructs. For $D_{2} Q_{15} K_{2}$, this width corresponds nearly exactly to the length of a fully extended strand of this 19-residue peptide, assuming a typical $\beta$-strand-like extension of around $3.5 \AA$ per residue. As mentioned, chemical shift data indicate that, at least in a considerable fraction of $\mathrm{D}_{2} \mathrm{Q}_{15} \mathrm{~K}_{2}$ molecules, two consecutive central glutamines must be in a standard extended $\beta$-sheet conformation, such that there cannot be a turn in the middle of these molecules. Additionally, wateredited ssNMR experiments indicate that Lys18 is exposed on the fibril surface (data not shown). While earlier investigations assumed the $\mathrm{D}_{2} \mathrm{Q}_{15} \mathrm{~K}_{2}$ peptide to fold into a hairpin-like structure in fibrillar aggregates, ${ }^{6,18,24}$ our data thus strongly suggest that it is rather present as a single extended strand. This leads to a model of $\mathrm{D}_{2} \mathrm{Q}_{15} \mathrm{~K}_{2}$ fibrillar aggregates as depicted in Fig. 7a.

With a similar $\beta$-strand length as in $\mathrm{D}_{2} \mathrm{Q}_{15} \mathrm{~K}_{2}$ and minimum fibril widths as found in electron micrographs, $\mathrm{GK}_{2} \mathrm{Q}_{38} \mathrm{~K}_{2}$ would form two $\beta$-strands and

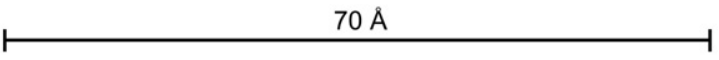

(a)

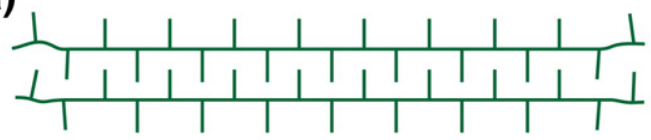

(b)

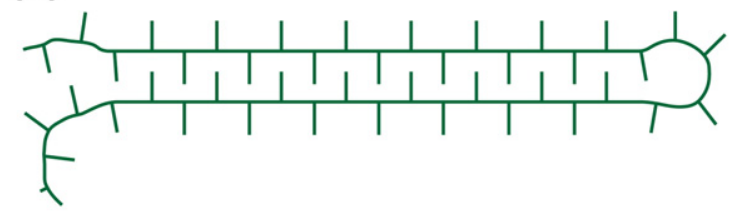

(c)
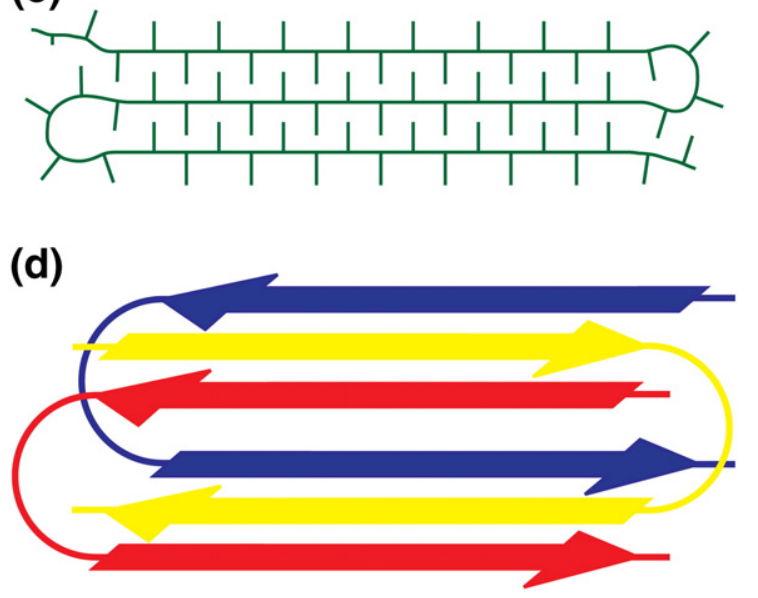

Fig. 7. Sketches of the proposed polyglutamine fibril model derived from ssNMR data. An approximate scale bar is indicated at the top. $(\mathrm{a}-\mathrm{c})$ Minimal repeat units of $\mathrm{D}_{2} \mathrm{Q}_{15} \mathrm{~K}_{2}$ (a), $\mathrm{GK}_{2} \mathrm{Q}_{38} \mathrm{~K}_{2}$ (b), and $\mathrm{GK}_{2} \mathrm{Q}_{54} \mathrm{~K}_{2}$ (c) fibrils, assuming similar $\beta$-strand lengths, viewed down the fibril axis. (d) Illustration of the superpleated antiparallel cross$\beta$ arrangement of monomers (here, corresponding to $\mathrm{GK}_{2} \mathrm{Q}_{38} \mathrm{~K}_{2}$ ) in polyglutamine fibrils, viewed down the fibril axis. Different monomers are shown in different colors. The fibril repeat unit consists of one $\mathrm{GK}_{2} \mathrm{Q}_{38} \mathrm{~K}_{2}$ molecule that forms two $\beta$-strands and contributes to two stacked $\beta$-sheets. 
$\mathrm{GK}_{2} \mathrm{Q}_{54} \mathrm{~K}_{2}$ would form three $\beta$-strands in a superpleated fold (Fig. $7 \mathrm{~b}$ and $\mathrm{c}$ ). Observed variations in fibril width, however, suggest not only lateral aggregation of individual protofilaments but also a polymorphic nature of the longer constructs in terms of $\beta$-strand length.

\section{Discussion}

We have investigated the structural arrangement of polyglutamine fibrils. Electron micrographs show that the length of the fibrillar aggregates increases with the length of the construct. Different fibril widths likely reflect different levels of lateral aggregation of protofilaments as well as different $\beta$-strand lengths. Water-edited spectroscopy shows that polyglutamine fibrils possess a dry fibrillar core of 70 to $80 \AA$ diameter or larger, depending on the construct. Our data are thus incompatible with water-filled, $\beta$-helical nanotubes, ${ }^{23}$ in agreement with molecular dynamics studies suggesting their limited stability. ${ }^{20,25}$ In an earlier ssNMR study, evidence for side-chain hydrogen bonding in the glutamine-rich yeast prion protein Ure2p was deduced from ${ }^{13} \mathrm{C}-{ }^{15} \mathrm{~N}$ dipolar couplings. ${ }^{26}$ Here, we have conducted a combined analysis of chemical shifts and through-space correlations suggesting that glutamine side chains in fibrillar polyglutamine aggregates are well ordered and tightly interdigitated as postulated by steric zipper models of amyloid fibrils. ${ }^{15,24}$ In particular, downfield shifts in glutamine side-chain proton resonances and through-space side chain-backbone correlations support the notion of glutamine side chains being hydrogen bonded within each sheet and interdigitated between sheets.

Local sequential and spatial organization appears to be similar in the different constructs. Despite rather large spectral linewidths, indicating structural variability, two distinct, approximately equally frequent main populations of glutamine residues constituting the $\beta$-sheet fibrillar core were identified in all samples. Residues belonging to these two populations are discernible by their chemical shift and can be found within individual monomers, clustered in stretches of several residues of one population. The findings of an increased spectral linewidth and of distinct chemical shift populations are in agreement with other studies on fibrils from asparagine- and glutamine-rich peptides. ${ }^{21,42,51}$ While residue groups with different chemical shifts have usually been assumed to constitute different fibrils in such samples, ${ }^{42}$ we demonstrate here that they can also occur within individual fibrils and monomers.

Through-space NHHC data and secondary chemical shifts reveal that both major glutamine chemical shift populations are in $\beta$-sheet conformations.
Distinct backbone chemical shift sets could reflect peptide segments that exhibit different backbone dihedral angles. Since $Q(1)$ and $Q(2)$ glutamines occur in a ratio of about 1:1, their dihedral angles may, for example, mutually compensate each other, thus reducing $\beta$-strand twist. Indeed, it has been demonstrated by cryo-EM and Fourier transform infrared spectroscopy that $\beta$-sheets in amyloid fibrils are more planar than those in globular proteins, which allows for the assembly of long filaments with little or no filament twist. ${ }^{52,53} \mathrm{~A}$ flat, planar $\beta$-sheet requires $\beta$-strands with only a small degree of strand twist along the polypeptide chain. Such flat $\beta$-strands have already been found or postulated for amyloid fibrils in X-ray and ssNMR studies, ${ }^{15,29,52,54,55}$ where they are assembled from residues with narrow dihedral angle distributions centered around the $\Psi=-\Phi$ diagonal of the Ramachandran plot.

To investigate a possible correlation between chemical shift population and backbone conformation of glutamine residues, we conducted an in silico grid search for glutamine backbone conformations with optimal agreement between predicted $^{56}$ and experimental chemical shifts (see Supplementary Methods). In addition, we searched the Biological Magnetic Resonance Data Bank database for glutamine residues with assignments corresponding to the more unusual population $\mathrm{Q}(1)$ values, for which no good fits could be obtained in the grid search (see Supplementary Methods for details). The dihedral angle distributions thus determined for the two $\beta$-sheet populations exhibit considerable scatter and overlap, preventing assignment of distinct backbone dihedral angle regions to the two residue groups (Fig. 5, Supporting Information). Nevertheless, the average values of the distributions are different, indicating that different average backbone dihedral angles may indeed partly account for the presence of the two groups of $\beta$-sheet glutamine residues.

Alternatively, the occurrence of two distinct sets of glutamine chemical shifts may be related to different side-chain rotameric states in the corresponding residues. It is well known that side-chain dihedral angles, especially $\chi_{1}$, can affect side-chain and backbone chemical shifts to an extent comparable to the shift differences observed here ${ }^{35,57-59}$ (see Fig. 6, Supporting Information). In a steric zipper arrangement, individual side-chain conformations are likely strongly constrained by side-chain hydrogen bonding and tight stacking of $\beta$-sheets. ${ }^{15,24}$ Using simulated annealing in $\mathrm{CNS}^{60}{ }^{60}$ we assembled a model exhibiting the core features of a polyglutamine fibril with a steric zipper arrangement (see Supplementary Methods). The model shows that enforcing close stacking of $\beta$-sheets and side-chain hydrogen bonding is indeed sufficient to yield a well-ordered side-chain arrangement (Fig. 7a, Supporting Information) with side-chain $\chi_{1}$ angles 
clustered in certain regions of dihedral angle space. In particular, it appears that the orientation of the side-chain carbonyl group can influence the $x_{1}$ angle (Fig. $7 \mathrm{~b}$ and c, Supplementary Information, and Supplementary Methods). This hypothesis thus offers an alternative explanation for the presence of populations of residues with distinct sets of chemical shifts. Certain side-chain hydrogen-bonding patterns may then lead to a clustering of similar $\chi_{1}$ angles along the peptide sequence, consistent with the clustering we observe for the chemical shift populations. This explanation would also well account for our findings that side-chain chemical shifts are distinct between the populations as well and that only few such shift populations are observed.

Interestingly, the two chemical shift populations can also be detected in polyglutamine aggregates that do not constitute bona fide fibrils, as well as in proline mutants (data not shown), where long-range order and side-chain hydrogen bonding appear less developed. Possibly, the steric constraints that lead to the development of preferred side-chain orientations are also present under these conditions, while the appearance of side chain-backbone cross-signals in ssNMR spectra may require a higher degree of order that is only achieved in fibrils and in the absence of $\beta$-strand-breaking residues. Direct measurement of side-chain torsion angles via chemical shift anisotropy correlation spectroscopy ${ }^{61,62}$ may help to clarify this issue.

Similar distinct sets of chemical shifts, also within individual fibrils, were found in a recent ssNMR study of fibrils formed by a peptide derived from the human islet amyloid polypeptide. ${ }^{63}$ There, larger chemical shift differences were attributed to residues with side chains pointing inside or outside the fibrils, respectively. However, this explanation would not account for our observations that $Q(1)$ and $Q(2)$ glutamines are clustered within the sequences of the peptides investigated here. Moreover, lateral aggregation of polyglutamine protofilaments, as suggested by EM data, should lead to a majority of side chains being buried within the fibrils. The 1:1 distribution of $Q(1)$ and $Q(2)$ glutamines can thus not be explained by side-chain exposure. Furthermore, the build-up characteristics of $Q(1)$ and $Q(2)$ in water-edited spectroscopy of $Q_{38}$ constructs are identical (data not shown). However, the side-chain rotameric state was also identified as a source for chemical shift differences in that study, ${ }^{63}$ in agreement with our hypotheses.

Another recent ssNMR study, published while our work was under review, investigated fibrils formed by the $\mathrm{N}$-terminal region of the huntingtin protein $\left(\mathrm{htt}^{\mathrm{NT}}\right) .{ }^{51}$ In excellent agreement with our results, glutamine residues in $\mathrm{htt}^{\mathrm{NT}}$ fibrils were also found to be in a rigid and hydrophobic fibrillar core as well as to give rise to two distinct sets of chemical shifts. Notably, this result was the same for fibrils formed by simple polyglutamine peptides as well as for $\mathrm{htt}^{\mathrm{NT}}$ fibrils. That study also found that the chemical shift populations are independent of sequence position and that an antiparallel arrangement of the $\beta$-sheets is likely. However, our study also explicitly investigates fibril structure and addresses the question of the occurrence of glutamine residues with different chemical shifts within single molecules and fibrils.

Our data indicate that $\mathrm{D}_{2} \mathrm{Q}_{15} \mathrm{~K}_{2}$ peptides aggregate as single extended $\beta$-strands. Fibrils of the longer constructs appear to contain $\beta$-strands of similar length; however, variations in fibril width suggest a polymorphic nature. The question of $\beta$-strand length in polyglutamine fibrils was also investigated in an earlier mutation study of polyglutamine peptides containing turn-inducing proline-glycine pairs. Such peptides aggregated as rapidly as polyglutamine homopolypeptides of equal length, provided the spacing of proline-glycine moieties still allowed for formation of $\beta$-strands at least seven residues in length. ${ }^{64}$ This agrees well with our ssNMR data, since the finding that $\mathrm{D}_{2} \mathrm{Q}_{15} \mathrm{~K}_{2}$ does not form a turn or bend indicates that $\beta$-strands in polyglutamine fibrils should be at least seven or eight residues long. However, in the absence of such mutations, longer $\beta$-strands appear to be the preferred arrangement of polyglutamine peptides in fibrils. This is indicated, on the one hand, by minimum fibril diameters observed in our EM data, which are always about 70-80 A. In addition, in a preliminary mutational study on $\mathrm{GK}_{2} \mathrm{Q}_{38} \mathrm{~K}_{2}$, we have introduced single proline mutations at positions that, according to our model, should be within long $\beta$-strands (residue 14 or 33 of the peptide sequence). These mutations should break $\beta$-strand secondary structure without necessarily enforcing turns. Fibril formation in these constructs was less efficient, and through-space backbone-side chain correlations indicative of a well-ordered side-chain arrangement were absent, indicating that these mutations indeed interfered with formation of the $\beta$-sheet fibrillar core (data not shown). However, another mutation study found reduced aggregation levels in proline mutants of polyglutamine peptides even if the proline residues were inserted at positions that should be within loop regions according to our model. ${ }^{65}$ Further mutational studies will be necessary to resolve this issue and delineate the exact influence of residue position and type on fibril formation. Possibly, however, these results simply indicate that there is not a single well-defined loop region shared by all molecules in polyglutamine fibrils, again highlighting the possibility of polymorphism in folding and aggregation of glutamine homopolypeptides.

Our data thus cannot provide a definitive answer to the question of $\beta$-strand length in the longer 
polyglutamine constructs. However, the conclusion that uninterrupted polyglutamine peptides form relatively long $\beta$-strands upon aggregation is well in line with recent data published while our manuscript was under review. ${ }^{66}$ For a $\mathrm{K}_{2} \mathrm{Q}_{23} \mathrm{~K}_{2}$ peptide, an aggregation nucleus consisting of four molecules was found, which was interpreted to comprise four extended strand peptides. ${ }^{66}$ Such an arrangement, which should translate to the mature fibril as well, would in principle allow for even longer $\beta$-strands.

Neighboring strands within $\beta$-sheets of all polyglutamine constructs analyzed appear to belong to different monomers. This finding is best explained by a superpleated $\beta$-sandwich-like organization ${ }^{49}$ in which individual monomers are folded in a plane orthogonal to the fibril axis and contribute strands to more than one $\beta$-sheet. A similar type of fold has also been postulated for several other types of amyloid, such as fibrils formed from the prion domains of Ure $2 p,{ }^{26}$ Sup $35 p,{ }^{22}$ and the amyloid $\beta$ peptide. ${ }^{29}$ In the case of amyloid $\beta$, a staggering of strands from different monomers has been assumed, ${ }^{67}$ which would also be consistent with our data. The aforementioned mutation study on polyglutamine peptides with interspersed proline-glycine residue pairs suggested the presence of hairpin-like turns, entailing monomers folded within the plane of one $\beta$-sheet. However, proline-glycine pairs could also be accommodated within $\beta$-bends connecting strands between different sheets. ${ }^{20,64}$

The N/CHHC data presented here indicate that the polyglutamine fibrils investigated consist mainly of antiparallel $\beta$-sheets, in line with structural studies on shorter peptides that were also observed to adopt an antiparallel organization. ${ }^{68-72}$ These findings may be a direct consequence of electrostatic interactions that favor an antiparallel arrangement of the charged peptide termini.

Apart from a superpleated $\beta$-sandwich structure, a triangular $\beta$-helical arrangement with a tightly packed hydrophobic core might be envisioned for polyglutamine fibrils, as suggested by some molecular dynamics studies. ${ }^{25,73}$ Such structures have been found in a large number of proteins ${ }^{74}$ and, similarly, in a recent ssNMR study of amyloid fibrils from the HET-s(218-289) yeast prion. ${ }^{31}$ The presence of random-coil-like population $\mathrm{Q}(3)$ chemical shifts in the spectra of $\mathrm{D}_{2} \mathrm{Q}_{15} \mathrm{~K}_{2}$ might be consistent with a triangular arrangement of this molecule. However, we rather attribute these signals to the loose, unstructured material visible in some electron micrographs. Triangular fibrils would have a smaller width (no more than about $30 \AA$ A) than observed in EM images (Fig. 1). Additionally, the side chain-backbone contacts seen in NHHC and HETCOR spectra are best explained by a regular pattern of interdigitated side chains from adjacent $\beta$-sheets, which is more difficult to achieve in a triangular arrangement of side chains with equal length, as in a homopolypeptide. A triangular $\beta$-helical structure would also not account for the $8.3 \AA$ reflection in fibril X-ray diffractograms. ${ }^{24}$ Finally, our data for the longer polyglutamine constructs indicate that neighboring strands within a $\beta$-sheet originate from different monomers arranged in an antiparallel fashion, which would be incompatible with $\beta$-helical models. Thus, we consider the antiparallel superpleated $\beta$-sandwich structure with tightly stacked sheets and interdigitated side chains to be most likely for the polyglutamine fibrils investigated here. This type of structure has also been shown to be stable in molecular dynamics simulations. ${ }^{19}$

Our data provide no clear indication for a structural basis of the toxicity limit of polyglutamine stretches around 37 residues. In agreement with a biophysical study, ${ }^{75}$ our ssNMR spectra indicate that the local structural organization of fibrillar aggregates from constructs of different lengths is highly similar, although EM micrographs demonstrate increasing long-range order with increasing monomer length. This may be related to the presence of more $\beta$-strands per monomer in the longer constructs, that is, a larger aggregation surface. With a $\beta$-strand length of around 15 residues as apparent for $\mathrm{D}_{2} \mathrm{Q}_{15} \mathrm{~K}_{2}$, about 40 residues would be the minimum length for a single polyglutamine peptide to assume a conformation as postulated here for the aggregated state, that is, two $\beta$-strands folded back on top of each other with interdigitating side chains. However, in view of the varying minimal lengths of polyglutamine stretches necessary for toxicity in different diseases, ${ }^{2}$ the conformational transition leading to toxicity may not only be accessible for glutamine repeats above the 37-residue limit. Length-dependent differences in thermodynamics or kinetics of the folding reaction might be sufficient to explain the different degrees of toxicity. ${ }^{75}$

While aggregation kinetics and, in the case of Huntington's disease, age of onset have been shown to correlate with glutamine repeat length, ${ }^{7}$ some studies have suggested that the toxic polyglutamine species are monomeric or oligomeric rather than fibrillar. ${ }^{8,9}$ This issue is still under debate, ${ }^{76}$ and it has been shown that polyglutamine aggregates can also have toxic effects on cells. ${ }^{5}$ Toxicity in monomers, however, seems to occur only after a conformational transition to a $\beta$-sheet-rich state. ${ }^{8}$ It is thus possible that toxic monomeric or oligomeric species of polyglutamine peptides exhibit similar structural features as the monomers within the fibrils investigated in this study. This notion would also agree with the claim that the rate-limiting step in aggregation is an unfavorable conformational transition within a single molecule ${ }^{7,77}$ and with our finding that the characteristic two $\beta$-sheet chemical shift 
populations also appear in small spherical or fibrillar aggregates that do not constitute fibrils. However, it is not known whether toxic monomeric or oligomeric polyglutamine species or the small aggregates in our study are on- or off-pathway to fully formed fibrils. The observation of toxicity in polyglutamine monomers and oligomers certainly points to the need for more detailed structural investigation of these species. In addition, recent evidence suggests that flanking and distant sequences in the host protein and the cellular context are crucial for toxicity and disease progression as well as aggregation mechanism. ${ }^{10,11,78,79}$ This means that a complete picture of polyglutamine aggregation and toxicity will likely not be obtained if only the polyglutamine stretches per se are considered.

Despite the presence of only a single dominant amino acid type and fibril polymorphism, a consistent structural view of polyglutamine fibrils emerges from our study. Our data indicate that the polyglutamine fibrils investigated here are antiparallel, superpleated cross- $\beta$ structures with $\beta$-sheets stacked tightly on top of each other. Experimental evidence supports a steric zipper arrangement with side chains hydrogen bonded along the fibril axis within each sheet and closely interdigitated between adjacent sheets. In contrast, $\beta$-helical structures, especially if assumed to be water filled, are disfavored. Our data provide novel insight into the structural organization of polyglutamine aggregates and provide the basis for further studies investigating, for example, monomeric and oligomeric polyglutamine species and effects of inhibitors of fibril formation.

\section{Materials and Methods}

\section{Peptide synthesis and expression}

For synthesis of the peptide $\mathrm{D}_{2} \mathrm{Q}_{15} \mathrm{~K}_{2}$ with residues Asp2, Gln3, Gln6, Gln9, Gln10, Gln17, and Lys18 uniformly ${ }^{13} \mathrm{C}^{15} \mathrm{~N}$-labeled, 9-fluorenylmethyloxycarbonyl (Fmoc)-protected amino acids Fmoc-Asp(t-butyl)-OH, Fmoc-Gln(trityl)-OH, and Fmoc-Lys(t-butyl)-OH were purchased from Merck Biosciences. Labeled amino acids were obtained from Cambridge Isotope Laboratories. $\mathrm{D}_{2} \mathrm{Q}_{15} \mathrm{~K}_{2}$ was synthesized on $217 \mathrm{mg}(0.05 \mathrm{mmol})$ TentaGel SRAM (Rapp Polymers) by manual solid-phase peptide synthesis with a 2-(1H-benzotriazole-1-yl)1,1,3,3-tetramethyluronium hexafluorophosphate activation protocol. The Fmoc group was removed by $20 \%$ piperidine $/ N, N$-dimethylformamide $(2 \times 10 \mathrm{~min})$. Unlabeled amino acids were coupled using 5 eq of amino acid, 5 eq of 2-(1H-benzotriazole-1-yl)-1,1,3,3-tetramethyluronium hexafluorophosphate, 5 eq of $N$-hydroxybenzotriazole, and $10 \mathrm{eq}$ of $N, N$-diisopropylethylamine with a coupling time of $30 \mathrm{~min}$. Labeled amino acids were coupled with 1.2 eq of amino acid, 1.3 eq of 2-(7-aza- $1 H_{-}$ benzotriazole-1-yl)-1,1,3,3-tetramethyluronium hexafluorophosphate and 2.6 eq of $N, N$-diisopropylethylamine and a coupling time of $18 \mathrm{~h}$. All washing steps after coupling and deprotection and all coupling steps were carried out with $N, N$-dimethylformamide. After the final Fmoc deprotection, the N-terminus was acetylated with acetic anhydride $\left(\mathrm{Ac}_{2} \mathrm{O}\right)$. The peptide was cleaved from the resin by treatment with trifluoroacetic acid (TFA)/ethanedithiol/triisopropylsilane/water (in a 85/5/5/5 ratio) for $6 \mathrm{~h}$. The crude peptide was precipitated with diethyl ether, washed, and lyophilized. The peptide was purified on a Jasco HPLC system and analyzed by electrospray ionization mass spectrometry.

The DNA sequences coding for the other polyglutamine constructs were assembled from six different oligonucleotides. The first oligonucleotide pair was flanked by a BamHI restriction site and encoded for a tobacco etch virus protease cleavage site. The middle part, which was translated into 15 glutamine repeats, was used to vary the length of the glutamine stretch. This oligonucleotide pair was phosphorylated with $\mathrm{T} 4$ polynucleotide kinase (Fermentas) for $2 \mathrm{~h}$ at $37^{\circ} \mathrm{C}$ to enable length variations during the subsequent hybridization. The last oligonucleotide pair encoded for eight glutamine residues and had both a stop codon and an EcoRI restriction site. The oligonucleotides were hybridized, whereas the ratio of the second oligonucleotide pair was varied, resulting in different lengths of the glutamine coding sequence with at least eight repeats. The hybridized constructs were cloned into the BamHI and EcoRI sites of a pGEX-2T plasmid vector (Amersham Pharmacia). The vector itself contained Schistosoma japonicum glutathione $S$-transferase to increase protein solubility during expression and to facilitate protein purification.

Uniformly ${ }^{13} \mathrm{C}^{15} \mathrm{~N}$-labeled polyglutamine constructs were recombinantly expressed in Escherichia coli strain B121 as glutathione $S$-transferase fusion proteins and purified by affinity chromatography using glutathione agarose beads (Amersham Pharmacia). Subsequent tobacco etch virus protease cleavage resulted in $\mathrm{GK}_{2} \mathrm{Q}_{38} \mathrm{~K}_{2}$ and $\mathrm{GK}_{2} \mathrm{Q}_{54} \mathrm{~K}_{2}$ constructs, which were solubilized as described elsewhere. ${ }^{80}$ Solubilized peptides were purified on a preparative RP-C18 HPLC column $(250 \mathrm{~mm} \times 20 \mathrm{~mm}$; Bishoff Chromatography) with a $5-70 \%$ acetonitrile gradient. Peptide masses were determined by electrospray ionization mass spectroscopy on an LCQ Advantage Max (Finnigan) operating in positive ion mode. Fractions containing the desired product were lyophilized and subsequently solubilized in water with $0.1 \%$ TFA for peptide fibrillization.

\section{Peptide fibrillization}

For fibrillization, either the lyophilized peptide $\left(\mathrm{D}_{2} \mathrm{Q}_{15} \mathrm{~K}_{2}\right)$ or the peptide in water with $0.1 \%$ TFA at pH $3\left(\mathrm{GK}_{2} \mathrm{Q}_{38} \mathrm{~K}_{2}, \mathrm{GK}_{2} \mathrm{Q}_{54} \mathrm{~K}_{2}\right)$ was dissolved in or transferred to Tris-buffered saline buffer (10 mM Tris/ $\mathrm{HCl}$ and $150 \mathrm{mM} \mathrm{NaCl}$ ) at $\mathrm{pH} 7.4$ in a concentration of $100 \mu \mathrm{M}$ and stored at $37{ }^{\circ} \mathrm{C}$ for 4 days. Alternatively, prolonged incubation for 12 weeks in $0.1 \%$ TFA was used $\left(\mathrm{GK}_{2} \mathrm{Q}_{54} \mathrm{~K}_{2}\right)$, which also leads to fibrillization. ${ }^{80}$ Then, aggregates were harvested by ultracentrifugation at $208,000 \mathrm{~g}$ for $1 \mathrm{~h}$. 


\section{Electron microscopy}

EM samples shown in Fig. 1 and Fig. 2 in Supplementary Information were evaluated with a CM 120 TEM instrument (FEI, Eindhoven, The Netherlands). Pictures were taken with a $2048 \times 2048$ TemCam 224A camera (TVIPS, Gauting, Germany) using 52,000×-fold magnification at $-1.15-\mu \mathrm{m}$ defocus. Amyloid fibers, resuspended from the pellet, were prepared on a glow-discharged carbon foil and stained with 1\% uranyl acetate.

\section{Solid-state NMR}

Experiments were conducted using 2.5-mm, 3.2-mm, or 4-mm triple-resonance ${ }^{1} \mathrm{H},{ }^{13} \mathrm{C},{ }^{15} \mathrm{~N}$ magic-angle-spinning (MAS) probeheads at static magnetic fields of 9.4, 14.1, $16.4,18.8$, or $20.0 \mathrm{~T}$ corresponding to proton resonance frequencies between 400 and $850 \mathrm{MHz}$ (Bruker Biospin, Karlsruhe, Germany). Effective sample temperatures were calibrated using nickelocene ${ }^{81}$ and varied from about $-10{ }^{\circ} \mathrm{C}$ for $\left({ }^{15} \mathrm{~N},{ }^{13} \mathrm{C}\right)$ correlation spectra at high field to about $+7{ }^{\circ} \mathrm{C}$ for $\left({ }^{13} \mathrm{C},{ }^{13} \mathrm{C}\right)$ correlation spectra. MAS frequencies used were $11 \mathrm{kHz}$ for $\left({ }^{15} \mathrm{~N},{ }^{13} \mathrm{C}\right)$ spectra and 11.2 or $12.5 \mathrm{kHz}$ (at an $800 \mathrm{MHz}$ proton resonance frequency) for $\left({ }^{13} \mathrm{C},{ }^{13} \mathrm{C}\right)$ spin diffusion spectra conducted under weak coupling conditions. ${ }^{45}$ Mixing times of 20, 150, or $500 \mathrm{~ms}$ were used to obtain intra-residue, sequential, or long-range $\left({ }^{13} \mathrm{C}^{13} \mathrm{C}\right)$ correlations, respectively. ${ }^{1} \mathrm{H}$ field strengths for $90^{\circ}$ pulses and SPINAL64 decoupling ${ }^{82}$ were around $80 \mathrm{kHz}$. Initial $\left({ }^{1} \mathrm{H},{ }^{13} \mathrm{C}\right)$ or $\left({ }^{1} \mathrm{H},{ }^{15} \mathrm{~N}\right) \mathrm{CP}$ times were set to $700 \mu \mathrm{s}$. $\left({ }^{15} \mathrm{~N},{ }^{13} \mathrm{C}\right)$ spectra were recorded using SPECIFIC-CP $\mathrm{CP}^{83,84}$ as the $\left({ }^{15} \mathrm{~N},{ }^{13} \mathrm{C}\right)$ transfer step, using contact times of 3-4.5 ms, followed by homonuclear $\left({ }^{13} \mathrm{C}^{13} \mathrm{C}\right)$ spin diffusion mixing of up to $50 \mathrm{~ms}$. N/ $\mathrm{CHHC}^{46,47}$ spectra used short CPs bracketing the longitudinal $\left({ }^{1} \mathrm{H},{ }^{1} \mathrm{H}\right)$ mixing block $\left[80-100 \mu\right.$ s for $\left({ }^{1} \mathrm{H}^{13} \mathrm{C}\right)$-CPs and $150 \mu \mathrm{s}$ for $\left({ }^{1} \mathrm{H},{ }^{15} \mathrm{~N}\right)$-CPs] to limit transfer to directly bonded nuclei. $\left({ }^{1} \mathrm{H},{ }^{1} \mathrm{H}\right)$ mixing times in N/CHHC spectra were 100 or $250 \mu \mathrm{s}$. Frequency-switched homonuclear LeeGoldburg ${ }^{33}$ decoupling at a ${ }^{1} \mathrm{H}$ field strength of $83 \mathrm{kHz}$ was used for the indirect ${ }^{1} \mathrm{H}$ evolution period of the HETCOR correlation spectra in Fig. 2. The proton dimension of these spectra was calibrated using standard ${ }^{1} \mathrm{H}_{-}{ }^{13} \mathrm{C}$ and ${ }^{1} \mathrm{H}_{-}{ }^{15} \mathrm{~N}$ HETCOR experiments as a reference. The CP times used for the ${ }^{1} \mathrm{H}-{ }^{15} \mathrm{~N}$ and ${ }^{1} \mathrm{H}-{ }^{13} \mathrm{C}$ FSLGHETCOR spectra shown in Fig. 2 were $40 \mu \mathrm{s}$ and $120 \mu \mathrm{s}$, respectively. Water-edited one-dimensional $\mathrm{CP}$ buildup experiments $^{40}$ were recorded on 400 or $850 \mathrm{MHz}$ instruments at 6.5 or $10 \mathrm{kHz}$ MAS and $+7^{\circ} \mathrm{C}$ effective sample temperature. A 3-ms Gaussian pulse and a $T_{2}$ filter containing two delays of $1 \mathrm{~ms}$ each were used for selective water excitation before longitudinal $\left({ }^{1} \mathrm{H},{ }^{1} \mathrm{H}\right)$ mixing and $\left({ }^{1} \mathrm{H}^{13} \mathrm{C}\right) \mathrm{CP}$ transfer of $700 \mu \mathrm{s}$. Two-dimensional spectra were typically acquired using 1280 data points in the direct dimension and 375,34 , or 25 data points in the indirect dimension of $\left({ }^{13} \mathrm{C},{ }^{13} \mathrm{C}\right),\left({ }^{15} \mathrm{~N},{ }^{13} \mathrm{C}\right)$, or NHHC correlation spectra, respectively, for measurements at an $800 \mathrm{MHz}$ proton resonance frequency. $\mathrm{CHHC}$ spectra were recorded using 1024 and 60 data points in direct and indirect dimensions, respectively, at a $600 \mathrm{MHz}$ proton resonance frequency. Spectra were processed in XWinNMR or Topspin (Bruker Biospin), using $1024 \times 1024$ processing data points, and analyzed with Sparky (T. D. Goddard and D. G. Kneller, SPARKY 3, University of California, San
Francisco). Evaluation of ssNMR water-edited buildups was performed as described elsewhere ${ }^{40}$ and is described in further detail in Supporting Information.

\section{Acknowledgements}

This work was supported by the Deutsche Forschungsgemeinschaft (Grant RA 1781/1-1), the Max Planck Society, the Fonds der chemischen Industrie (Grant 684052), and Netherlands Organisation for Scientific Research (Grant 700.26.121). R.S. thanks the Deutsche Forschungsgemeinschaft graduate school 782 "Spectroscopy and Dynamics of Molecular Coils and Aggregates" for a PhD fellowship.

\section{Supplementary Data}

Supplementary data associated with this article can be found, in the online version, at doi:10.1016/ j.jmb.2011.06.045

\section{References}

1. Bates, G. (2003). Huntingtin aggregation and toxicity in Huntington's disease. Lancet, 361, 1642-1644.

2. Orr, H. T. (2001). Beyond the Qs in the polyglutamine diseases. Genes Dev. 15, 925-932.

3. Wanker, E. E. (2000). Protein aggregation and pathogenesis of Huntington's disease: mechanisms and correlations. Biol. Chem. 381, 937-942.

4. Gusella, J. F. \& MacDonald, M. E. (2000). Molecular genetics: unmasking polyglutamine triggers in neurodegenerative disease. Nat. Rev., Neurosci. 1, 109-115.

5. Yang, W., Dunlap, J. R., Andrews, R. B. \& Wetzel, R. (2002). Aggregated polyglutamine peptides delivered to nuclei are toxic to mammalian cells. Hum. Mol. Genet. 11, 2905-2917.

6. Chen, S. M., Berthelier, V., Hamilton, J. B., O'Nuallain, B. \& Wetzel, R. (2002). Amyloid-like features of polyglutamine aggregates and their assembly kinetics. Biochemistry, 41, 7391-7399.

7. Chen, S. M., Ferrone, F. A. \& Wetzel, R. (2002). Huntington's disease age-of-onset linked to polyglutamine aggregation nucleation. Proc. Natl Acad. Sci. USA, 99, 11884-11889.

8. Nagai, Y., Inui, T., Popiel, H. A., Fujikake, N., Hasegawa, K., Urade, Y. et al. (2007). A toxic monomeric conformer of the polyglutamine protein. Nat. Struct. Mol. Biol. 14, 332-340.

9. Takahashi, T., Kikuchi, S., Katada, S., Nagai, Y., Nishizawa, M. \& Onodera, O. (2008). Soluble polyglutamine oligomers formed prior to inclusion body formation are cytotoxic. Hum. Mol. Genet. 17, 345-356.

10. Thakur, A. K., Jayaraman, M., Mishra, R., Thakur, M., Chellgren, V. M., Byeon, I. J. L. et al. (2009). Polyglutamine disruption of the huntingtin exon $1 \mathrm{~N}$ terminus triggers a complex aggregation mechanism. Nat. Struct. Mol. Biol. 16, 380-389. 
11. Truant, R., Atwal, R. S., Desmond, C., Munsie, L. \& Tran, T. (2008). Huntington's disease: revisiting the aggregation hypothesis in polyglutamine neurodegenerative diseases. FEBS J. 275, 4252-4262.

12. Shorter, J. \& Lindquist, S. (2005). Prions as adaptive conduits of memory and inheritance. Nat. Rev., Genet. 6, 435-450.

13. Michelitsch, M. D. \& Weissman, J. S. (2000). A census of glutamine/asparagine-rich regions: implications for their conserved function and the prediction of novel prions. Proc. Natl Acad. Sci. USA, 97, 11910-11915.

14. Ader, C., Frey, S., Maas, W., Schmidt, H. B., Görlich, D. \& Baldus, M. (2010). Amyloid-like interactions within nucleoporin FG hydrogels. Proc. Natl Acad. Sci. USA, 107, 6281-6285.

15. Nelson, R., Sawaya, M. R., Balbirnie, M., Madsen, A. O., Riekel, C., Grothe, R. et al. (2005). Structure of the cross-beta spine of amyloid-like fibrils. Nature, $435,773-778$.

16. Sawaya, M. R., Sambashivan, S., Nelson, R., Ivanova, M. I., Sievers, S. A., Apostol, M. I. et al. (2007). Atomic structures of amyloid cross-beta spines reveal varied steric zippers. Nature, 447, 453-457.

17. Perutz, M. F. (1995). Glutamine repeats as polar zippers - their role in inherited neurodegenerative disease. Mol. Med. 1, 718-721.

18. Sharma, D., Shinchuk, L. M., Inouye, H., Wetzel, R. \& Kirschner, D. A (2005). Polyglutamine homopolymers having 8-45 residues form slablike beta-crystallite assemblies. Proteins: Struct., Funct., Bioinf. 61, 398-411.

19. Esposito, L., Paladino, A., Pedone, C. \& Vitagliano, L. (2008). Insights into structure, stability, and toxicity of monomeric and aggregated polyglutamine models from molecular dynamics simulations. Biophys. J. 94, 4031-4040.

20. Zanuy, D., Gunasekaran, K., Lesk, A. M. \& Nussinov, R. (2006). Computational study of the fibril organization of polyglutamine repeats reveals a common motif identified in beta-helices. J. Mol. Biol. 358, 330-345.

21. Baxa, U., Wickner, R. B., Steven, A. C., Anderson, D. E., Marekov, L. N., Yau, W. M. et al. (2007). Characterization of beta-sheet structure in Ure2p(189) yeast prion fibrils by solid-state nuclear magnetic resonance. Biochemistry, 46, 13149-13162.

22. Shewmaker, F., Wickner, R. B. \& Tycko, R. (2006). Amyloid of the prion domain of Sup35p has an inregister parallel beta-sheet structure. Proc. Natl Acad. Sci. USA, 103, 19754-19759.

23. Perutz, M. F., Finch, J. T., Berriman, J. \& Lesk, A. (2002). Amyloid fibers are water-filled nanotubes. Proc. Natl Acad. Sci. USA, 99, 5591-5595.

24. Sikorski, P. \& Atkins, E. (2005). New model for crystalline polyglutamine assemblies and their connection with amyloid fibrils. Biomacromolecules, 6, 425-432.

25. Stork, M., Giese, A., Kretzschmar, H. A. \& Tavan, P. (2005). Molecular dynamics simulations indicate a possible role of parallel beta-helices in seeded aggregation of poly-Gln. Biophys. J. 88, 2442-2451.

26. Chan, J. C. C., Oyler, N. A., Yau, W. M. \& Tycko, R. (2005). Parallel beta-sheets and polar zippers in amyloid fibrils formed by residues 10-39 of the yeast prion protein Ure2p. Biochemistry, 44, 10669-10680.
27. Heise, H., Hoyer, W., Becker, S., Andronesi, O. C., Riedel, D. \& Baldus, M. (2005). Molecular-level secondary structure, polymorphism, and dynamics of fulllength alpha-synuclein fibrils studied by solid-state NMR. Proc. Natl Acad. Sci. USA, 102, 15871-15876.

28. Kloepper, K. D., Zhou, D. H., Li, Y., Winter, K. A., George, J. M. \& Rienstra, C. M. (2007). Temperaturedependent sensitivity enhancement of solid-state NMR spectra of alpha-synuclein fibrils. J. Biomol. NMR, 39, 197-211.

29. Petkova, A. T., Ishii, Y., Balbach, J. J., Antzutkin, O. N., Leapman, R. D., Delaglio, F. et al. (2002). A structural model for Alzheimer's beta-amyloid fibrils based on experimental constraints from solid state NMR. Proc. Natl Acad. Sci. USA, 99, 16742-16747.

30. Andronesi, O. C., von Bergen, M., Biernat, J., Seidel, K., Griesinger, C., Mandelkow, E. et al. (2008). Characterization of Alzheimer's-like paired helical filaments from the core domain of tau protein using solid-state NMR spectroscopy. J. Am. Chem. Soc. 130, 5922-5928.

31. Wasmer, C., Lange, A., Van Melckebeke, H., Siemer, A. B., Riek, R. \& Meier, B. H. (2008). Amyloid fibrils of the HET-s(218-289) prion form a beta solenoid with a triangular hydrophobic core. Science, 319, 1523-1526.

32. Loquet, A., Bousset, L., Gardiennet, C., Sourigues, Y., Wasmer, C., Habenstein, B. et al. (2009). Prion fibrils of Ure2p assembled under physiological conditions contain highly ordered, natively folded modules. J. Mol. Biol. 394, 108-118.

33. Lee, M. \& Goldburg, W. I. (1965). Nuclear-magneticresonance line narrowing by a rotating rf field. Phys. Rev. 140, 1261.

34. Wishart, D. S., Sykes, B. D. \& Richards, F. M. (1991). Relationship between nuclear magnetic resonance chemical shift and protein secondary structure. J. Mol. Biol. 222, 311-333.

35. Shen, Y. \& Bax, A. (2007). Protein backbone chemical shifts predicted from searching a database for torsion angle and sequence homology. J. Biomol. NMR, 38, 289-302.

36. Moon, S. \& Case, D. A. (2007). A new model for chemical shifts of amide hydrogens in proteins. $J$. Biomol. NMR, 38, 139-150.

37. Ulrich, E. L., Akutsu, H., Doreleijers, J. F., Harano, Y., Ioannidis, Y. E., Lin, J. et al. (2007). BioMagResBank. Nucleic Acids Res. 36, D402-D408.

38. Lesage, A., Gardiennet, C., Loquet, A., Verel, R., Pintacuda, G., Emsley, L. et al. (2008). Polarization transfer over the water-protein interface in solids. Angew. Chem., Int. Ed. Engl. 47, 5851-5854.

39. Kumashiro, K. K., Schmidt-Rohr, K., Murphy, O. J., Ouellette, K. L., Cramer, W. A. \& Thompson, L. K. (1998). A novel tool for probing membrane protein structure: solid-state NMR with proton spin diffusion and X-nucleus detection. J. Am. Chem. Soc. 120, 5043-5051.

40. Ader, C., Schneider, R., Seidel, K., Etzkorn, M., Becker, S. \& Baldus, M. (2009). Structural rearrangements of membrane proteins probed by water-edited solidstate NMR spectroscopy. J. Am. Chem. Soc. 131, 170-176.

41. Heise, H. (2008). Solid-state NMR spectroscopy of amyloid proteins. ChemBioChem, 9, 179-189. 
42. van der Wel, P. C. A., Lewandowski, J. R. \& Griffin, R. G. (2007). Solid-state NMR study of amyloid nanocrystals and fibrils formed by the peptide GNNQQNY from yeast prion protein Sup35p. J. Am. Chem. Soc. 129, 5117-5130.

43. Luca, S., Filippov, D. V., van Boom, J. H., Oschkinat, H., de Groot, H. J. M. \& Baldus, M. (2001). Secondary chemical shifts in immobilized peptides and proteins: a qualitative basis for structure refinement under magic angle spinning. J. Biomol. NMR, 20, 325-331.

44. Andronesi, O. C., Becker, S., Seidel, K., Heise, H., Young, H. S. \& Baldus, M. (2005). Determination of membrane protein structure and dynamics by magicangle-spinning solid-state NMR spectroscopy. J. Am. Chem. Soc. 127, 12965-12974.

45. Seidel, K., Lange, A., Becker, S., Hughes, C. E., Heise, H. \& Baldus, M. (2004). Protein solid-state NMR resonance assignments from $\left({ }^{13} \mathrm{C},{ }^{13} \mathrm{C}\right)$ correlation spectroscopy. Phys. Chem. Chem. Phys. 6, 5090-5093.

46. Lange, A., Luca, S. \& Baldus, M. (2002). Structural constraints from proton-mediated rare-spin correlation spectroscopy in rotating solids. J. Am. Chem. Soc. 124, 9704-9705.

47. Lange, A., Seidel, K., Verdier, L., Luca, S. \& Baldus, M. (2003). Analysis of proton-proton transfer dynamics in rotating solids and their use for 3D structure determination. J. Am. Chem. Soc. 125, 12640-12648.

48. Seidel, K., Etzkorn, M., Heise, H., Becker, S. \& Baldus, M. (2005). High-resolution solid-state NMR studies on uniformly $\left[{ }^{13} \mathrm{C},{ }^{15} \mathrm{~N}\right]$-labeled ubiquitin. ChemBioChem, 6, 1638-1647.

49. Kajava, A. V., Baxa, U., Wickner, R. B. \& Steven, A. C. (2004). A model for Ure2p prion filaments and other amyloids: the parallel superpleated beta-structure. Proc. Natl Acad. Sci. USA, 101, 7885-7890.

50. Wüthrich, K. (1986). NMR of Proteins and Nucleic Acids. Wiley Interscience, NY.

51. Sivanandam, V. N., Jayaraman, M., Hoop, C. L., Kodali, R., Wetzel, R. \& van der Wel, P. C. (2011). The aggregation-enhancing huntingtin $\mathrm{N}$-terminus is helical in amyloid fibrils. J. Am. Chem. Soc. 133, $4558-4566$.

52. Zandomeneghi, G., Krebs, M. R. H., McCammon, M. G. \& Fandrich, M. (2004). FTIR reveals structural differences between native beta-sheet proteins and amyloid fibrils. Protein Sci. 13, 3314-3321.

53. Jimenez, J. L., Nettleton, E. J., Bouchard, M., Robinson, C. V., Dobson, C. M. \& Saibil, H. R. (2002). The protofilament structure of insulin amyloid fibrils. Proc. Natl Acad. Sci. USA, 99, 9196-9201.

54. Jaroniec, C. P., MacPhee, C. E., Astrof, N. S., Dobson, C. M. \& Griffin, R. G. (2002). Molecular conformation of a peptide fragment of transthyretin in an amyloid fibril. Proc. Natl Acad. Sci. USA, 99, 16748-16753.

55. Jaroniec, C. P., MacPhee, C. E., Bajaj, V. S., McMahon, M. T., Dobson, C. M. \& Griffin, R. G. (2004). Highresolution molecular structure of a peptide in an amyloid fibril determined by magic angle spinning NMR spectroscopy. Proc. Natl Acad. Sci. USA, 101, 711-716.

56. Neal, S., Nip, A. M., Zhang, H. \& Wishart, D. S. (2003). Rapid and accurate calculation of protein ${ }^{1} \mathrm{H},{ }^{13} \mathrm{C}$ and ${ }^{15} \mathrm{~N}$ chemical shifts. J. Biomol. NMR, 26, 215-240.
57. Xu, X. P. \& Case, D. A. (2001). Automated prediction of ${ }^{15} \mathrm{~N},{ }^{13} \mathrm{C}$ (alpha), ${ }^{13} \mathrm{C}$ (beta) and ${ }^{13} \mathrm{C}^{\prime}$ chemical shifts in proteins using a density functional database. $J$. Biomol. NMR, 21, 321-333.

58. Xu, X. P. \& Case, D. A. (2002). Probing multiple effects on ${ }^{15} \mathrm{~N},{ }^{13} \mathrm{C}$ alpha, ${ }^{13} \mathrm{C}$ beta, and ${ }^{13} \mathrm{C}^{\prime}$ chemical shifts in peptides using density functional theory. Biopolymers, 65, 408-423.

59. Villegas, M. E., Vila, J. A. \& Scheraga, H. A. (2007). Effects of side-chain orientation on the ${ }^{13} \mathrm{C}$ chemical shifts of antiparallel beta-sheet model peptides. J. Biomol. NMR, 37, 137-146.

60. Brunger, A. T., Adams, P. D., Clore, G. M., DeLano, W. L., Gros, P., Grosse-Kunstleve, R. W. et al. (1998). Crystallography \& NMR system: a new software suite for macromolecular structure determination. Acta Crystallogr., Sect. D: Biol. Crystallogr. 54, 905-921.

61. Wei, Y., Lee, D. K. \& Ramamoorthy, A. (2001). Solidstate ${ }^{13} \mathrm{C}$ NMR chemical shift anisotropy tensors of polypeptides. J. Am. Chem. Soc. 123, 6118-6126.

62. Yao, X. L. \& Hong, M. (2002). Determination of C alpha chemical shift tensor orientation in peptides by dipolar-modulated chemical shift recoupling NMR spectroscopy. J. Am. Chem. Soc. 124, 2730-2738.

63. Nielsen, J. T., Bjerring, M., Jeppesen, M. D., Pedersen, R. O., Pedersen, J. M., Hein, K. L. et al. (2009). Unique identification of supramolecular structures in amyloid fibrils by solid-state NMR spectroscopy. Angew. Chem., Int. Ed. 48, 2118-2121.

64. Thakur, A. K. \& Wetzel, R. (2002). Mutational analysis of the structural organization of polyglutamine aggregates. Proc. Natl Acad. Sci. USA, 99, 17014-17019.

65. Popiel, H. A., Nagai, Y., Onodera, O., Inui, T., Fujikake, N., Urade, Y. et al. (2004). Disruption of the toxic conformation of the expanded polyglutamine stretch leads to suppression of aggregate formation and cytotoxicity. Biochem. Biophys. Res. Commun. 317, 1200-1206.

66. Kar, K., Jayaraman, M., Sahoo, B., Kodali, R. \& Wetzel, R. (2011). Critical nucleus size for disease-related polyglutamine aggregation is repeat-length dependent. Nat. Struct. Mol. Biol. 18, 328-336.

67. Petkova, A. T., Yau, W. M. \& Tycko, R. (2006). Experimental constraints on quaternary structure in Alzheimer's beta-amyloid fibrils. Biochemistry, 45, 498-512.

68. Lansbury, P. T., Costa, P. R., Griffiths, J. M., Simon, E. J., Auger, M., Halverson, K. J. et al. (1995). Structural model for the beta-amyloid fibril based on interstrand alignment of an antiparallel sheet comprising a Cterminal peptide. Nat. Struct. Biol. 2, 990-998.

69. Petkova, A. T., Buntkowsky, G., Dyda, F., Leapman, R. D., Yau, W. M. \& Tycko, R. (2004). Solid state NMR reveals a $\mathrm{pH}$-dependent antiparallel beta-sheet registry in fibrils formed by a beta-amyloid peptide. J. Mol. Biol. 335, 247-260.

70. Bu, Z. M., Shi, Y., Callaway, D. J. E. \& Tycko, R. (2007). Molecular alignment within beta-sheets in A beta(1423) fibrils: solid-state NMR experiments and theoretical predictions. Biophys. J. 92, 594-602.

71. Sikorski, P., Atkins, E. D. T. \& Serpell, L. C. (2003). Structure and texture of fibrous crystals formed by Alzheimer's A beta(11-25) peptide fragment. Structure, 11, 915-926. 
72. Gordon, D. J., Balbach, J. J., Tycko, R. \& Meredith, S. C. (2004). Increasing the amphiphilicity of an amyloidogenic peptide changes the beta-sheet structure in the fibrils from antiparallel to parallel. Biophys. J. 86, 428-434.

73. Khare, S. D., Ding, F., Gwanmesia, K. N. \& Dokholyan, N. V (2005). Molecular origin of polyglutamine aggregation in neurodegenerative diseases. Plos Comput. Biol. 1, 230-235.

74. Jenkins, J. \& Pickersgill, R. (2001). The architecture of parallel beta-helices and related folds. Prog. Biophys. Mol. Biol. 77, 111-175.

75. Klein, F. A. C., Pastore, A., Masino, L., Zeder-Lutz, G., Nierengarten, H., Oulad-Abdeighani, M. et al. (2007). Pathogenic and non-pathogenic polyglutamine tracts have similar structural properties: towards a lengthdependent toxicity gradient. J. Mol. Biol. 371, 235-244.

76. Ross, C. A. \& Tabrizi, S. J. (2011). Huntington's disease: from molecular pathogenesis to clinical treatment. Lancet Neurol. 10, 83-98.

77. Bhattacharyya, A. M., Thakur, A. K. \& Wetzel, R. (2005). Polyglutamine aggregation nucleation: thermodynamics of a highly unfavorable protein folding reaction. Proc. Natl Acad. Sci. USA, 102, 15400-15405.
78. Dehay, B. \& Bertolotti, A. (2006). Critical role of the proline-rich region in Huntingtin for aggregation and cytotoxicity in yeast. J. Biol. Chem. 281, 35608-35615.

79. Bhattacharyya, A., Thakur, A. K., Chellgren, V. M., Thiagarajan, G., Williams, A. D., Chellgren, B. W. et al. (2006). Oligoproline effects on polyglutamine conformation and aggregation. J. Mol. Biol. 355, 524-535.

80. Chen, S. M. \& Wetzel, R. (2001). Solubilization and disaggregation of polyglutamine peptides. Protein Sci. 10, 887-891.

81. Heise, H., Kohler, F. H. \& Xie, X. L. (2001). Solid-state NMR spectroscopy of paramagnetic metallocenes. $J$. Magn. Reson. 150, 198-206.

82. Fung, B. M., Khitrin, A. K. \& Ermolaev, K. (2000). An improved broadband decoupling sequence for liquid crystals and solids. J. Magn. Reson. 142, 97-101.

83. Baldus, M., Petkova, A. T., Herzfeld, J. \& Griffin, R. G. (1998). Cross polarization in the tilted frame: assignment and spectral simplification in heteronuclear spin systems. Mol. Phys. 95, 1197-1207.

84. Petkova, A. T., Baldus, M., Belenky, M., Hong, M., Griffin, R. G. \& Herzfeld, J. (2003). Backbone and side chain assignment strategies for multiply labeled membrane peptides and proteins in the solid state. J. Magn. Reson. 160, 1-12. 